\title{
Actinidia chinensis Planch.: A Review of Chemistry and Pharmacology
}

\author{
Xirui $\mathrm{He}^{1 *}$, Jiacheng Fang ${ }^{2}$, Xufei Chen ${ }^{2}$, Zefeng Zhao ${ }^{2}$, Yongsheng $\mathrm{Li}^{3}$, Yibing Meng ${ }^{3}$ \\ and Linhong Huang ${ }^{3}$
}

${ }^{1}$ Department of Bioengineering, Zhuhai Campus Zunyi Medical University, Zhuhai, China, ${ }^{2}$ The College of Life Sciences, Northwest University, Xi'an, China, ${ }^{3}$ Honghui Hospital, Xi'an Jiaotong University, Xi'an, China

OPEN ACCESS

Edited by:

Monique S.J. Simmonds, Royal Botanic Gardens,

Kew, United Kingdom

Reviewed by: Marinella De Leo, University of Pisa, Italy Jianping Chen,

Shenzhen Traditional Chinese Medicine Hospital,

China

${ }^{*}$ Correspondence:

Xirui $\mathrm{He}$

xiruihe6105194@163.com

Specialty section:

This article was submitted to

Ethnopharmacology,

a section of the journal

Frontiers in Pharmacology

Received: 10 April 2019 Accepted: 27 September 2019 Published: 30 October 2019

Citation:

He X, Fang J, Chen X, Zhao Z, Li Y,

Meng $Y$ and Huang L (2019) Actinidia chinensis Planch.: A Review of Chemistry and Pharmacology.

Front. Pharmacol. 10:1236. doi: 10.3389/fphar.2019.01236
Actinidia chinensis Planch. (A. chinensis), commonly known as Chinese kiwifruit, is a China native fruit, which becomes increasingly popular due to attractive economic, nutritional, and health benefits properties. The whole plant including fruits, leaves, vines, and roots of $A$. chinensis are used mainly as food or additive in food products and as folk medicine in China. It is a good source of triterpenoids, polyphenols, vitamin $\mathrm{C}$, carbohydrate, amino acid, and minerals. These constituents render the $A$. chinensis with a wide range of pharmacological properties including antitumor, antioxidant, antiinflammatory, immunoregulatory, hypolipemic, antidiabetic, and cardiovascular protective activities, suggesting that it may possibly be value in the prevention and treatment of pathologies associated to cancer, oxidative stress, and aging. This minireview provides a brief knowledge about the recent advances in chemistry, biological activities, utilization, and storage of Chinese kiwifruit. Future research directions on how to better use of this crop are suggested.

Keywords: Actinidia chinensis, nutritional composition, chemistry, pharmacological properties, antitumor, antioxidant

\section{INTRODUCTION}

Actinidia chinensis Planch. (A. chinensis), commonly known as "Chinese kiwifruit" (English), “中华狝猴桃” (Chinese), and characterized by excessive vegetative vigor, is a woody perennial, deciduous, and functionally dioecious medicinal plant in the family Actinidiaceae (Flora of China, 2007; The Plant List, 2013). It is native to China and has been cultivated in New Zealand, United States, Greece, Italy, Chile, France, Japan, and Korea (Li and Zhu, 2017; Ma et al., 2017). In China, they are mainly distributed in temperate to warm-temperate zones such as Shaanxi, Gansu, Henan, Guangdong, Guangxi, Fujian, Guizhou, Yunnan, Sichuan, as well as the middle and lower reaches of the Yangtze River basin, especially in Yiling district in Yichang city, Hubei province (Figure 1) (Flora of China, 2007). There are 13 A. chinensis cultivars, especially "Hongyang," "Jintao," and "Huayou," are developed for commercial production in China (Sharon, 2016), and more than three ones such as "Sungold," "Charm," and "Hort16A" developed in New Zealand (Henare, 2016) (Table 1).

There are two varieties accepted by The Plant List that include A. chinensis and A. chinensis var. setosa H.L.Li (The Plant List, 2013). The fruit of A. chinensis is the largest one in Actinidia genus, and it has the greatest economic, medicinal, and edible significance in terms of production and utilization. Its relevant pictures are showed in Figure 2. Generally, Chinese kiwifruit with a crosssectional radius of about $3 \mathrm{~cm}$ is oval-shaped densely covered with yellowish-brown hairs. The flesh color of fruit skin is green to yellow, and the average fruit weight is $20-120 \mathrm{~g}$. The fruit is a tasty, nutritious food that can be eaten fresh directly. Today, a range of kiwifruit processed products with 


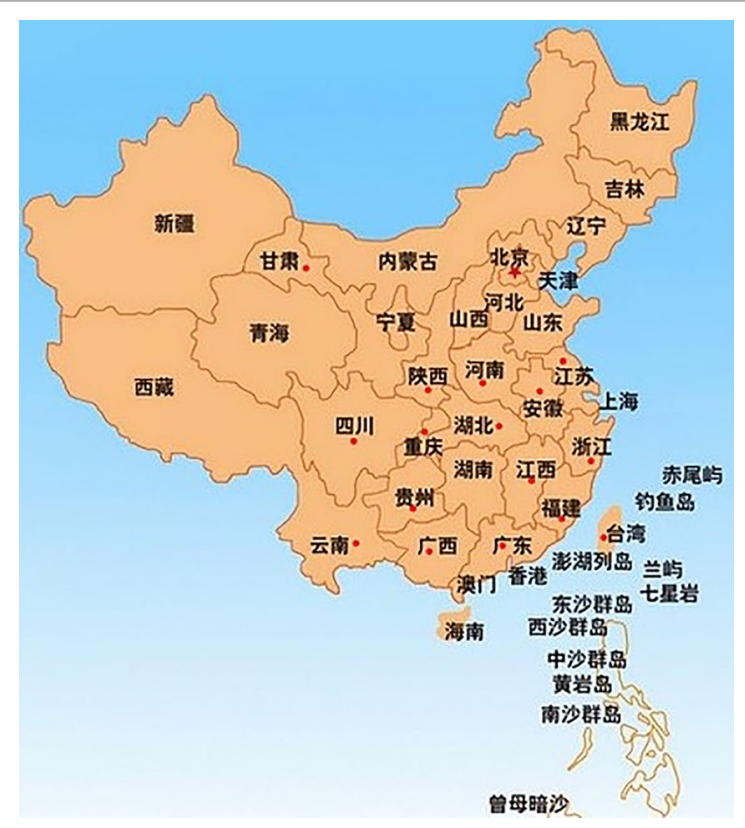

FIGURE 1 | The red spots in the map depicted the main region of A. chinensis distribution in China (Flora of China, 2007; https://www.newasp.net/soft/105257.html).

Abbreviations: A549, Human alveolar basal epithelial cells; ABTS, 2'-azinobis (3-ethylbenzthiazoline-6-sulfonic acid; ACE, Angiotensin converting enzyme; ALT, Alanine aminotransferase; AST, Aspartate aminotransferase; CE, Catechin equivalents; COX-2, Cyclooxygenase-2; CYP2C9, Cytochrome P450 2C9; CYP2D6, Cytochrome P450 2D6; CYP3A4, Cytochrome P450 3A4; CYPs, Cytochrome P450; DLX2, Distal-less-like homeobox protein; DPPH, 2,2-diphenyl1-picrylhydrazyl; DW, Dry weight; EP3, Prostaglandin E2 receptor subtype 3; ERK1/2, Extracellular regulated protein kinases; FAS, Fatty acid synthase; FDW, Freeze-dried weight; FRAP, Ferric ion reducing antioxidant power; FW, flesh weight; GABA-BZD, $\gamma$-aminobutyric acid-benzodiazepine; GAE, Gallic acid equivalents; GC-MS, Gas chromatography-mass spectrometer; GSH, Glutathione; HaCaT, Human immortalized keratinocytes; HCC, Hepatocellular carcinoma; HDL-C, High density lipoprotein cholesterol; HEK 293, Human embryonic kidney 293 cells; HepG2, Liver hepatocellular cells; HO·, Hydroxyl radical; HOMA-IR, Homeostasis model assessment for insulin resistance; HPLC, Highperformance liquid chromatography; $\mathrm{IC}_{50}$, Half maximal inhibitory concentration; IL-1 $\beta$, Interleukin-1 $\beta$; IL-6, Interleukin-6; iNOS, Inducible nitric oxide synthase; Keap1, Kelch-like ECH-associated protein 1; LC-MS/MS, Liquid chromatographytandem mass spectrometry; LDL-C, Low density lipoprotein cholesterol; LoVo, Colorectal cancer cell line; MCF-7, Human breast adenocarcinoma cell line; MDA, Malondialdehyde; MIC, Minimum inhibitory concentration; NCI-H460, Large cell lung cancer cells; NF-кB, Nuclear factor-kappa B; NO, Nitric oxide; Nrf2, Nuclear factor (erythroid-derived 2)-like 2; ORAC, Oxygen radical absorbance capacity; P-388, Mouse leukemia cells; PGC1- $\alpha$, Peroxisome proliferator-activated receptor gamma coactivator 1-alpha; PI3K/AKT, Phosphatidylinositol 3-kinase/ protein kinase B; PPAR- $\gamma$, Peroxisome proliferators-activated receptors; PRDM16, PR domain containing 16; QE, Quercetin equivalents; RAW 264.7, Mouse leukaemic monocyte macrophage cell line; SK-OV-3, Human ovarian epithelial cancer cells; SOD, Superoxide dismutase; TC, Total cholesterol; TG, Triglyceride; TNF- $\alpha$, Tumor necrosis factor alpha; UCP1, Uncoupling protein 1; UPLC-MS/ MS, Ultra-high-performance liquid chromatography-tandem mass spectrometry; UPLC-QqQ-MS/MS, Ultra-high-performance hydrophilic interaction liquid chromatography-triple quadrupole tandem mass; UPLCTOF/MS, Ultra-performance liquid chromatography quadrupole-time of flight mass spectrometry; USDA, United States Department of Agriculture; UV/Vis, Ultraviolet-visible spectroscopy; VCE, Vitamin C equivalents; VEGFR2/Src/FAK, Vascular endothelial growth factor receptor $2 / \mathrm{Src} /$ focal adhesion kinase. the attractive eating quality and nutritional benefits has been developed including juice, preserved fruit, yogurt, wine, canned fruit, dried kiwi slices, fruit vegetable juice drinks, milk beverage, and vinegar. Apart from being a food and natural health product, the whole plant (fruits, branches and leaves, vines and roots) of A. chinensis has been used as traditional folk medicine in China (He et al., 2017; Wei et al., 2018; Fang et al., 2019). The ripe kiwifruit, tastes sweet and sour, acts on the spleen, stomach, and kidney meridians, has improving properties on dyspepsia, loss of appetite, and vomiting. The branches and leaves have been used to treat arthronalgia, bleeding, empyrosis, and ulcer. The vine has appetizing, heat clearing, and wind-dampness dispelling effects and is used to treat indigestion, aundice, and urolithiasis. The root and bark of $A$. chinensis taste bitter and astringent, and they have various medical effects such as wind and heat dispelling, blood circulation improving, and detumescence properties, and are used for the treatment of rheumatoid arthritis, bruises, furuncle, swelling, filariasis, hepatitis, and dysentery (Xie, 1975). However, people with weak spleen and stomach should be cautious in taking A. chinensis (Xie, 1975). To date, only very few modern studies have been done on potential toxic and side effects of $A$. chinensis, which should be highlighted in future research.

The principal chemical composition of the whole plant of A. chinensis include polyphenol, triterpenoids and derivatives, carotenoids, polysaccharides, amino acids, vitamins, essential oils, and microelements. (Papunidze et al., 2001; Chang and Case, 2005; Ma et al., 2017; Wang et al., 2017; Twidle et al., 2018). Among these ingredients, the main bioactive constituents are phenolic compounds, triterpenes, and the major nutritional composition are vitamin $\mathrm{C}$, vitamin $\mathrm{E}$, dietary fiber, and microelements, which make up a relatively 
TABLE 1 |A. chinensis cultivars developed for commercial production (Henare, 2016).

\begin{tabular}{|c|c|c|c|c|c|}
\hline Origin country & Cultivar & Fruit shape & Avg. weight & Fruit skin & Fruit flesh \\
\hline \multirow[t]{13}{*}{ China } & Cuiyu (Liangmei No. 1) & Ovoid & $90 \mathrm{~g}$ & Greenish brown with short hairs & Green \\
\hline & Wuzhi No. 3 (Wuzhi 81-36) & Ellipsoid & $85 \mathrm{~g}$ & Dark green with soft hairs & Bright green \\
\hline & $\begin{array}{l}\text { Chuhong (Panda }{ }^{\mathrm{TM}} \\
\text { Forest Red Kiwi) }\end{array}$ & Long ellipsoid & $80 \mathrm{~g}$ & Dark green and hairless & $\begin{array}{l}\text { Green with red flesh around white } \\
\text { core }\end{array}$ \\
\hline & Qihong & Cylindric & $100 \mathrm{~g}$ & Green with sparse or absent hairs & Light green to yellow \\
\hline & Hongyang (Red Sun, RS1) & Obovoid & $60-70 \mathrm{~g}$ & $\begin{array}{l}\text { Dark green or greenish brown } \\
\text { with fine hairs }\end{array}$ & $\begin{array}{l}\text { Green-yellow to yellow, circle of red } \\
\text { around white core }\end{array}$ \\
\hline & $\begin{array}{l}\text { Jintao (C6, WIB-C6, } \\
\left.\text { Jingold }{ }^{\mathrm{TM}}\right)\end{array}$ & Long cylindric & $90 \mathrm{~g}$ & Yellow with brown hairs & Green-yellow to orange-yellow \\
\hline & $\begin{array}{l}\text { Huayou (Panda }{ }^{\mathrm{TM}} \text { Golden } \\
\text { Kiwi) }\end{array}$ & Ellipsoid & $90 \mathrm{~g}$ & Ellipsoid & Light green to yellow \\
\hline & $\begin{array}{l}\text { Ganmi No. } 1 \text { (Zaoxian } \\
\text { No. 1, FT-79-5) }\end{array}$ & Cylindric & $85 \mathrm{~g}$ & $\begin{array}{l}\text { Green-brown to pale brown with } \\
\text { soft hairs }\end{array}$ & Greenish-yellow to yellow \\
\hline & $\begin{array}{l}\text { Ganmi No. } 3 \\
\text { (Jinfeng, FT 79-3) }\end{array}$ & Ellipsoid & $80-90 \mathrm{~g}$ & $\begin{array}{l}\text { Yellow-brown or dark brown with } \\
\text { short, fine hairs }\end{array}$ & Yellow \\
\hline & Jinyan & Cylindrical & $100-110 \mathrm{~g}$ & Yellow brown with short, fine hairs & Yellow \\
\hline & $\begin{array}{l}\text { Ganmi No. } 2 \text { (Kuimi, FY } \\
79-1 \text { ) }\end{array}$ & Apple shaped & $100 \mathrm{~g}$ & $\begin{array}{l}\text { Green-brown to dark brown with } \\
\text { fine hairs }\end{array}$ & Yellow-green to yellow \\
\hline & Hort16A & Ovoid & $95-100 \mathrm{~g}$ & Green-brown to brown with soft hairs & Yellow-green to bright yellow \\
\hline & Wanhong & Cylindrical & $110-140 \mathrm{~g}$ & Green-brown with rare hairs & Yellow-green to bright yellow \\
\hline \multirow[t]{3}{*}{ New Zealand } & Charm (Zespri® ${ }^{\circledR}$ Charm) & Ovoid & & Brown with soft hairs & Yellow \\
\hline & Sungold (Zespri ${ }^{\circledR}$ Sungold) & & & Brown with smooth skin & Yellow \\
\hline & $\begin{array}{l}\text { Hort16A (Zespri } \\
\text { Gold, Earligold) }\end{array}$ & Ovoid & $95-100 g$ & $\begin{array}{l}\text { Green-brown to brown with soft } \\
\text { hairs }\end{array}$ & Yellow-green to bright yellow \\
\hline Italy & Soreli (Ac 171.76) & Oblong & $>100 \mathrm{~g}$ & Brown with sparse hairs & Yellow \\
\hline Japan & Sanuki Gold & Squat & $160-180 \mathrm{~g}$ & Brown with soft hairs & Bright yellow \\
\hline
\end{tabular}

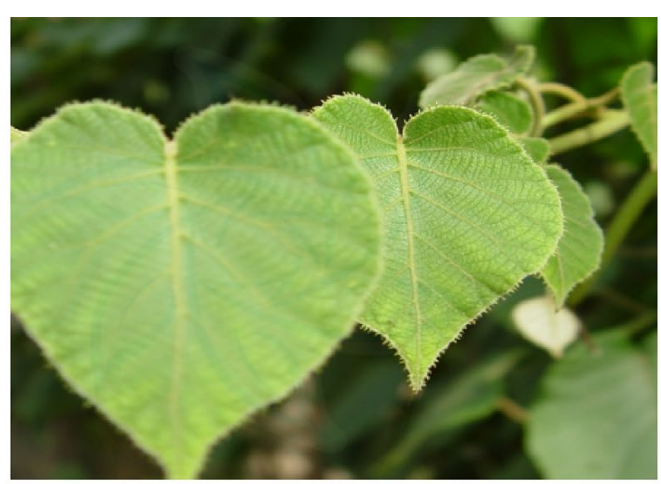

Leaves

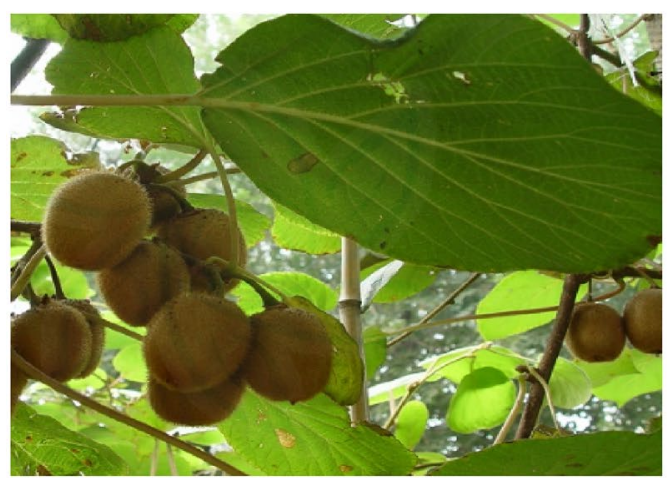

Fruit on the vine

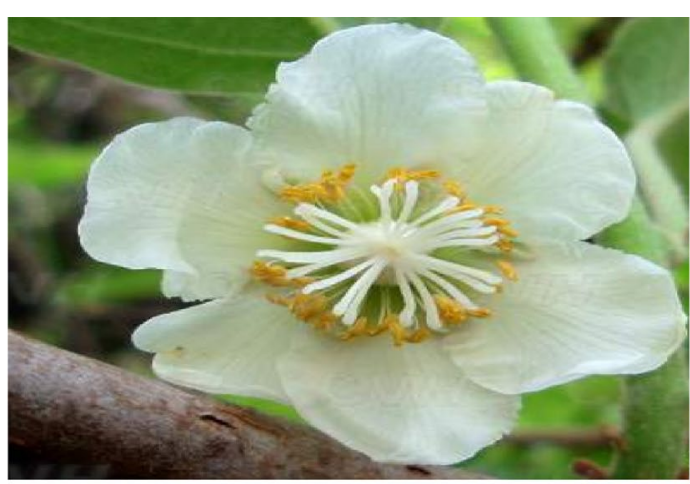

Flowers

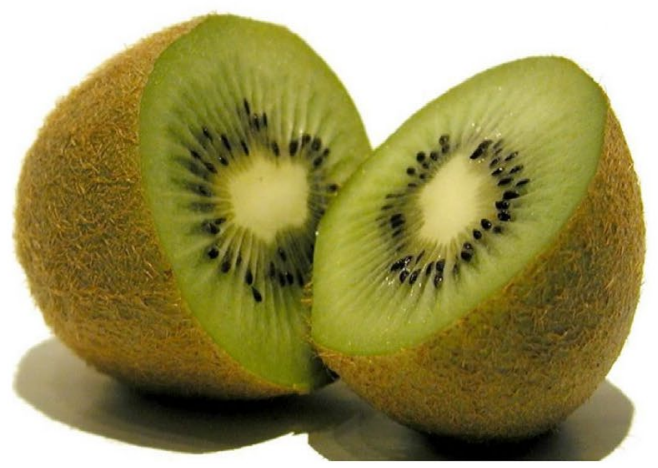

Kiwifruit and its cross-section, showing seeds

FIGURE 2 | The leaves, flowers, vines, and fruits of $A$. chinensis. 
significant share of the daily value (Table 2). Pharmacological results have revealed various promising bioactivities to $A$. chinensis including antitumor, antioxidant, anti-inflammatory, antimicrobial, immunoregulatory, hypolipemic and antidiabetic, cardiovascular protective, hypnotic effects, and ACE inhibitory activities (Deng et al., 2013; Niu et al., 2016; Sun et al., 2017; Xia et al., 2017; Deng et al., 2018; Hou et al., 2018; Fang et al., 2019). Much of these bioactivities of $A$. chinensis are consistent with those observed in traditional folk medicine. More importantly, A. chinensis showed significantly antitumor and antioxidant properties, and these effects could be depended on the presence of a range of triterpenoids, polysaccharide, and phenolic compounds (Chang and Case, 2005; Wei et al., 2018; Fang et al., 2019). However, the information on the chemical and biological activities of $A$. chinensis is scattered. In this review, we intend to systematically summarize the recent advances in nutritional composition, chemistry, and biological activities of A. chinensis

TABLE 2 | Nutritional composition of Zespri ${ }^{\circledR}$ sun-gold kiwifruit.

\begin{tabular}{|c|c|c|c|}
\hline Nutrient & Unit & $\begin{array}{l}\text { Kiwifruit } \\
81 \mathrm{~g}\end{array}$ & $\begin{array}{c}\text { Value per } \\
100 \mathrm{~g}\end{array}$ \\
\hline \multicolumn{4}{|l|}{ Proximates } \\
\hline Water & $g$ & 66.78 & 82.44 \\
\hline Energy & kcal & 51 & 63 \\
\hline Protein & $g$ & 0.83 & 1.02 \\
\hline Total lipid (fat) & $g$ & 0.23 & 0.28 \\
\hline Carbohydrate, by difference & $g$ & 12.79 & 15.79 \\
\hline Fiber, total dietary & $g$ & 1.1 & 1.4 \\
\hline Sugars, total & $g$ & 9.96 & 12.3 \\
\hline \multicolumn{4}{|l|}{ Minerals } \\
\hline Calcium, Ca & $\mathrm{mg}$ & 14 & 17 \\
\hline Iron, Fe & mg & 0.17 & 0.21 \\
\hline Magnesium, Mg & mg & 10 & 12 \\
\hline Phosphorus, $\mathrm{P}$ & mg & 20 & 25 \\
\hline Potassium, K & $\mathrm{mg}$ & 255 & 315 \\
\hline Sodium, $\mathrm{Na}$ & $\mathrm{mg}$ & 2 & 3 \\
\hline Zinc, Zn & $\mathrm{mg}$ & 0.06 & 0.08 \\
\hline \multicolumn{4}{|l|}{ Vitamins } \\
\hline Vitamin C, total ascorbic acid & $\mathrm{mg}$ & 130.7 & 161.3 \\
\hline Thiamin & $\mathrm{mg}$ & 0.000 & 0.000 \\
\hline Riboflavin & $\mathrm{mg}$ & 0.060 & 0.074 \\
\hline Niacin & $\mathrm{mg}$ & 0.187 & 0.231 \\
\hline Vitamin B-6 & mg & 0.064 & 0.079 \\
\hline Folate, DFE & $\mu g$ & 25 & 31 \\
\hline Vitamin B-12 & $\mu g$ & 0.06 & 0.08 \\
\hline Vitamin A, RAE & $\mu g$ & 1 & 1 \\
\hline Vitamin A, IU & IU & 19 & 23 \\
\hline Vitamin E (alpha-tocopherol) & $\mathrm{mg}$ & 1.13 & 1.40 \\
\hline Vitamin D (D2 + D3) & $\mu g$ & 0.0 & 0.0 \\
\hline Vitamin D & IU & 0 & 0 \\
\hline Vitamin K (phylloquinone) & $\mu \mathrm{g}$ & 4.9 & 6.1 \\
\hline \multicolumn{4}{|l|}{ Lipids } \\
\hline Fatty acids, total saturated & $g$ & 0.053 & 0.065 \\
\hline $\begin{array}{l}\text { Fatty acids, total } \\
\text { monounsaturated }\end{array}$ & $g$ & 0.019 & 0.023 \\
\hline $\begin{array}{l}\text { Fatty acids, total } \\
\text { polyunsaturated }\end{array}$ & $g$ & 0.090 & 0.111 \\
\hline Fatty acids, total trans & $g$ & 0 & 0 \\
\hline Cholesterol & $\mathrm{mg}$ & 0 & 0 \\
\hline
\end{tabular}

Source: USDA Food Composition Databases, https://ndb.nal.usda.gov/ndb/Accessed on April, 2018. and also provide future research directions for better utilize and develop it as a sustainable crop.

\section{CHEMICAL COMPOSITION}

\section{Nutritional Composition}

Chinese kiwifruit, known as the "king of fruits," is a fruit with high-pulp juices, thick flesh, delicious taste, and rich nutrition and has a higher commercial and economic value. It is a rich source of various nutrients including vitamins, carbohydrate, sugar, minerals, amino acids, protein, fatty acids (e.g., linoleic acid), and carotenoids. Table 2 lists the nutritional composition of sun-gold kiwifruit reported from the USDA Food Composition Database (United States Department of Agriculture, USDA Food Composition Databases, 2018). Table 3 shows the chemical content of A. chinensis fruit (Chang and Case, 2005; Cui et al., 2007; Zhou et al., 2009; Xu et al., 2010; He et al., 2014; He et al., 2015a; He et al., 2015b; Xu et al., 2016; Twidle et al., 2018; Sivakumaran et al., 2018; Wei et al., 2018; Zhang et al., 2018). Of particular note, nutritional composition in kiwifruit is vitamin $\mathrm{C}$ $(1.61 \mathrm{mg} / \mathrm{g})$ and minerals $\mathrm{K}(3.15 \mathrm{mg} / \mathrm{g})$. The average vitamin $\mathrm{C}$ content of Huayou, Jintao, Ganmi-1, Ganmi-2, Ganmi-3, Wuzhi3 , and Cuiyu cultivated in China are 1.59, 1.49, 0.86, 1.34, 0.97, 2.88 , and $1.18 \mathrm{mg} / \mathrm{g}$, respectively. Meanwhile, the vitamin C in SunGold was $1.61 \mathrm{mg} / \mathrm{g}$ edible flesh, followed by other varieties Sweet Green (1.5 mg/g) and green "Hayward" (0.85 mg/g) (Sivakumaran et al., 2018). Especially, the vitamin C content in kiwifruit is higher than that determined in lemon, orange, strawberry, and grapefruits (Ma et al., 2017).

The data show evidence that the sun-gold kiwifruit is high in carbohydrate $(15.79 \%)$ and sugars $(12.3 \%)$. The total starches contents were found for outer pericarp and core tissues ranged from $38.6 \%$ to $51.8 \%$ and $34.6 \%$ to $40.7 \%$ DW in three harvesting A. chinensis varieties, and the starches in core have higher amylose content (20.7\%-23.3\%) and enzyme susceptibility. However, the crystallinity degree, granule size, and gelatinization parameters of starches in core are somewhat lower (Li and Zhu, 2017). The kiwifruit peel contains a higher total pectin content $(3.7 \%-4.2 \%)$ than that of pulp (1.6\%-2.1\%) (Meng et al., 2017). Xia et al. (2017) analyzed the polysaccharide from Hongyang using water extraction, followed by column chromatography, high performance gel permeation chromatography, HPLC, and Fourier transform infrared spectroscopy. The results indicated that the polysaccharide of Hogyang fruit consisted of the following monosaccharides: D-galactose (25.45\%), D-galacturonic acid (25.25\%), L-arabinose (20.51\%), L-rhamnose (17.78\%), D-glucose (6.14\%), D-mannose (2.13\%), D-xylose $(1.03 \%)$, D-glucuronic acid $(0.97 \%)$, and D-fucose (0.74\%). These studies confirmed the utilization potential of Chinese kiwifruit as an incredibly healthy food and loaded with important nutrients and health benefits for human consumption.

Kiwifruit contained 18 free amino acids. Briefly, the total essential amino acid contents in Jintao, Hongyang, Huayou, and Hort16A cultivated in China are 2.59, 1.55, 2.0, and $2.09 \mathrm{mg} / \mathrm{g} \mathrm{FW}$, whereas the total essential amino acid in Hort16A cultivated in New Zealand was $2.06 \mathrm{mg} / \mathrm{g}$ FW. 
TABLE 3 | The nutritional composition or phytochemicals content of $A$. chinensis fruit.

\begin{tabular}{|c|c|c|c|c|c|c|}
\hline Composition & $\begin{array}{l}\text { Cultivar } \\
\text { location }\end{array}$ & Genotype & Method & Plant part & Content & Ref. \\
\hline $\begin{array}{l}\text { Vitamin C (ascorbic } \\
\text { acid) }\end{array}$ & Colomicta & A. chinensis & HPLC & Ripe fruits & $\begin{array}{l}0.82 \mathrm{mg} / \mathrm{g} \mathrm{FW} ; 4.34 \\
\mathrm{mg} / \mathrm{g} \mathrm{DW}\end{array}$ & Kvesitadze et al., 2001 \\
\hline Vitamin C & Cardinal & A. chinensis & HPLC & Ripe fruits & $\begin{array}{l}0.74 \mathrm{mg} / \mathrm{g} \mathrm{FW} ; 4.30 \\
\mathrm{mg} / \mathrm{g} \mathrm{DW}\end{array}$ & Kvesitadze et al., 2001 \\
\hline Vitamin C & Bruno & A. chinensis & HPLC & Ripe fruits & $\begin{array}{l}0.76 \mathrm{mg} / \mathrm{g} \text { FW; } 4.28 \\
\mathrm{mg} / \mathrm{g} \mathrm{DW}\end{array}$ & Kvesitadze et al., 2001 \\
\hline Vitamin C & Monti & A. chinensis & HPLC & Ripe fruits & $\begin{array}{l}0.76 \mathrm{mg} / \mathrm{g} \mathrm{FW} ; 4.33 \\
\mathrm{mg} / \mathrm{g} \mathrm{DW}\end{array}$ & Kvesitadze et al., 2001 \\
\hline Vitamin C & Purpuria & A. chinensis & HPLC & Ripe fruits & $\begin{array}{l}0.78 \mathrm{mg} / \mathrm{g} \mathrm{FW} ; 4.27 \\
\mathrm{mg} / \mathrm{g} \mathrm{DW}\end{array}$ & Kvesitadze et al., 2001 \\
\hline Vitamin C & Gaivard & A. chinensis & HPLC & Ripe fruits & $\begin{array}{l}0.72 \mathrm{mg} / \mathrm{g} F W ; 4.14 \\
\mathrm{mg} / \mathrm{g} \mathrm{DW}\end{array}$ & Kvesitadze et al., 2001 \\
\hline Vitamin C & Gaivard & A. chinensis & HPLC & Skin & $\begin{array}{l}0.21 \mathrm{mg} / \mathrm{g} \mathrm{FW} ; 0.63 \\
\mathrm{mg} / \mathrm{g} \mathrm{DW}\end{array}$ & Kvesitadze et al., 2001 \\
\hline Vitamin C & Gaivard & A. chinensis & HPLC & Pulp & $\begin{array}{l}0.85 \mathrm{mg} / \mathrm{g} \text { FW; } 4.75 \\
\mathrm{mg} / \mathrm{g} \mathrm{DW}\end{array}$ & Kvesitadze et al., 2001 \\
\hline Vitamin C & Gaivard & A. chinensis & HPLC & Core & $\begin{array}{l}0.48 \mathrm{mg} / \mathrm{g} F W ; 2.67 \\
\mathrm{mg} / \mathrm{g} \mathrm{DW}\end{array}$ & Kvesitadze et al., 2001 \\
\hline Vitamin C & Gaivard & A. chinensis & HPLC & Fresh juice & $\begin{array}{l}0.55 \text { mg/g FW; } 3.44 \\
\text { mg/g DW }\end{array}$ & Kvesitadze et al., 2001 \\
\hline Vitamin C & Gaivard & A. chinensis & HPLC & Juice after $24 \mathrm{~h}$ & $\begin{array}{l}0.55 \text { mg/g FW; } 3.44 \\
\mathrm{mg} / \mathrm{g} \mathrm{DW}\end{array}$ & Kvesitadze et al., 2001 \\
\hline Vitamin C & Shaanxi, China & Huayou & $\begin{array}{l}\text { 2,6-dichloroindophenol } \\
\text { titration method }\end{array}$ & Ripe fruits & 1.59 mg/g FW & Ma et al., 2017 \\
\hline $\begin{array}{l}\text { Total starch content } \\
\text { in tissue }\end{array}$ & Pukekohe & $\begin{array}{l}\text { Zespri }^{\circledR} \text { SunGold } \\
\text { Kiwifruit }\end{array}$ & Total starch assay kit & Outer pericarp & $38.6 \% \mathrm{DW}$ & Li and Zhu, 2017 \\
\hline $\begin{array}{l}\text { Total starch content } \\
\text { in tissue }\end{array}$ & Auckland & Gold9 & Total starch assay kit & Outer pericarp & $51.8 \%$ DW & Li and Zhu, 2017 \\
\hline $\begin{array}{l}\text { Total starch content } \\
\text { in tissue }\end{array}$ & New Zealand & Hort16A & Total starch assay kit & Outer pericarp & $44.8 \%$ DW & Li and Zhu, 2017 \\
\hline $\begin{array}{l}\text { Apparent amylose } \\
\text { content }\end{array}$ & Pukekohe & $\begin{array}{l}\text { Zespri }^{\circledR} \text { SunGold } \\
\text { Kiwifruit }\end{array}$ & Total starch assay kit & Outer pericarp & $27.5 \% \mathrm{DW}$ & Li and Zhu, 2017 \\
\hline $\begin{array}{l}\text { Apparent amylose } \\
\text { content }\end{array}$ & Auckland & Gold9 & Total starch assay kit & Outer pericarp & $24.5 \% \mathrm{DW}$ & Li and Zhu, 2017 \\
\hline $\begin{array}{l}\text { Apparent amylose } \\
\text { content }\end{array}$ & New Zealand & Hort16A & Total starch assay kit & Outer pericarp & $25.3 \%$ DW & Li and Zhu, 2017 \\
\hline True amylose content & Pukekohe & $\begin{array}{l}\text { Zespri }^{\circledR} \text { SunGold } \\
\text { Kiwifruit }\end{array}$ & Total starch assay kit & Outer pericarp & $17.8 \%$ DW & Li and Zhu, 2017 \\
\hline True amylose content & Auckland & Gold9 & Total starch assay kit & Outer pericarp & $15.7 \% \mathrm{DW}$ & Li and Zhu, 2017 \\
\hline True amylose content & New Zealand & Hort16A & Total starch assay kit & Outer pericarp & $15.5 \%$ DW & Li and Zhu, 2017 \\
\hline Total dietary fibre & New Zealand & Hort 16A & Megazyme method & puree & $34.1 \mathrm{mg} / \mathrm{g} \mathrm{FW}$ & Yuliarti et al., 2008 \\
\hline Total dietary fibre & New Zealand & Hort 16A & Megazyme method & Skin and cores & $13.84 \%$ DW & Yuliarti et al., 2008 \\
\hline Insoluble dietary fibre & New Zealand & Hort 16A & Megazyme method & Puree & $26.1 \mathrm{mg} / \mathrm{g} \mathrm{FW}$ & Yuliarti et al., 2008 \\
\hline Insoluble dietary fibre & New Zealand & Hort 16A & Megazyme method & Skin and cores & $11.39 \%$ DW & Yuliarti et al., 2008 \\
\hline Soluble dietary fibre & New Zealand & Hort 16A & Megazyme method & Puree & $8 \mathrm{mg} / \mathrm{g} \mathrm{FW}$ & Yuliarti et al., 2008 \\
\hline Soluble dietary fibre & New Zealand & Hort 16A & Megazyme method & Skin and cores & $2.45 \% \mathrm{DW}$ & Yuliarti et al., 2008 \\
\hline $\begin{array}{l}\text { Nonstarch } \\
\text { polysaccharide }\end{array}$ & New Zealand & gold kiwifruit & Acid extraction & Pomace & $77.59 \%$ DW & Yuliarti et al., 2015a \\
\hline $\begin{array}{l}\text { Nonstarch } \\
\text { polysaccharide }\end{array}$ & New Zealand & gold kiwifruit & Acid extraction & $\begin{array}{l}\text { Early-harvested } \\
\text { Fruits }\end{array}$ & $69.14 \%$ DW & Yuliarti et al., 2015b \\
\hline $\begin{array}{l}\text { Nonstarch } \\
\text { polysaccharide }\end{array}$ & New Zealand & gold kiwifruit & Acid extraction & $\begin{array}{l}\text { Main-harvested } \\
\text { fruits }\end{array}$ & $64.49 \%$ DW & Yuliarti et al., 2015b \\
\hline $\begin{array}{l}\text { Nonstarch } \\
\text { polysaccharide }\end{array}$ & New Zealand & gold kiwifruit & Water extraction & Pomace & $79.16 \%$ DW & Yuliarti et al., 2015a \\
\hline $\begin{array}{l}\text { Nonstarch } \\
\text { polysaccharide }\end{array}$ & New Zealand & gold kiwifruit & Water extraction & $\begin{array}{l}\text { Early-harvested } \\
\text { fruits }\end{array}$ & $60.74 \%$ DW & Yuliarti et al., 2015b \\
\hline $\begin{array}{l}\text { Nonstarch } \\
\text { polysaccharide }\end{array}$ & New Zealand & gold kiwifruit & Water extraction & $\begin{array}{l}\text { Main-harvested } \\
\text { fruits }\end{array}$ & $63.77 \%$ DW & Yuliarti et al., 2015b \\
\hline $\begin{array}{l}\text { Nonstarch } \\
\text { polysaccharide }\end{array}$ & New Zealand & gold kiwifruit & Enzymatic extraction & Pomace & $80.12 \%$ DW & Yuliarti et al., 2015a \\
\hline $\begin{array}{l}\text { Nonstarch } \\
\text { polysaccharide }\end{array}$ & New Zealand & gold kiwifruit & Enzymatic extraction & $\begin{array}{l}\text { Early-harvested } \\
\text { fruits }\end{array}$ & $39.21 \% \mathrm{DW}$ & Yuliarti et al., 2015b \\
\hline
\end{tabular}


TABLE 3 | Continued

\begin{tabular}{|c|c|c|c|c|c|c|}
\hline Composition & $\begin{array}{l}\text { Cultivar } \\
\text { location }\end{array}$ & Genotype & Method & Plant part & Content & Ref. \\
\hline $\begin{array}{l}\text { Nonstarch } \\
\text { polysaccharide }\end{array}$ & New Zealand & gold kiwifruit & Enzymatic extraction & $\begin{array}{l}\text { Main-harvested } \\
\text { fruits }\end{array}$ & $64.02 \%$ DW & Yuliarti et al., 2015b \\
\hline Total free amino acids & Shaanxi, China & Hort16A & $\begin{array}{l}\text { Hitachi L-8900 amino } \\
\text { acid analyzer }\end{array}$ & Ripe fruits & $8.31 \mathrm{mg} / \mathrm{g} \mathrm{FW}$ & Ma et al., 2017 \\
\hline Total free amino acids & New Zealand & Hort16A & $\begin{array}{l}\text { Hitachi L-8900 amino } \\
\text { acid analyzer }\end{array}$ & Ripe fruits & $8.01 \mathrm{mg} / \mathrm{g} \mathrm{FW}$ & Ma et al., 2017 \\
\hline Total free amino acids & Shaanxi, China & Huayou & $\begin{array}{l}\text { Hitachi L-8900 amino } \\
\text { acid analyzer }\end{array}$ & Ripe fruits & $7.15 \mathrm{mg} / \mathrm{g} \mathrm{FW}$ & Ma et al., 2017 \\
\hline $\begin{array}{l}\text { Total essential amino } \\
\text { acids }\end{array}$ & Shaanxi, China & Huayou & $\begin{array}{l}\text { Hitachi L-8900 amino } \\
\text { acid analyzer }\end{array}$ & Ripe fruits & $1.55 \mathrm{mg} / \mathrm{g} \mathrm{FW}$ & Ma et al., 2017 \\
\hline $\begin{array}{l}\text { Total essential amino } \\
\text { acids }\end{array}$ & Shaanxi, China & Hort16A & $\begin{array}{l}\text { Hitachi L-8900 amino } \\
\text { acid analyzer }\end{array}$ & Ripe fruits & $2.09 \mathrm{mg} / \mathrm{g} \mathrm{FW}$ & Ma et al., 2017 \\
\hline $\begin{array}{l}\text { Total essential amino } \\
\text { acids }\end{array}$ & New Zealand & Hort16A & $\begin{array}{l}\text { Hitachi L-8900 amino } \\
\text { acid analyzer }\end{array}$ & Ripe fruits & $2.06 \mathrm{mg} / \mathrm{g} \mathrm{FW}$ & Ma et al., 2017 \\
\hline $\begin{array}{l}\text { Nonessential amino } \\
\text { acids }\end{array}$ & New Zealand & Hort16A & $\begin{array}{l}\text { Hitachi L-8900 amino } \\
\text { acid analyzer }\end{array}$ & Ripe fruits & $5.95 \mathrm{mg} / \mathrm{g} \mathrm{FW}$ & Ma et al., 2017 \\
\hline $\begin{array}{l}\text { Nonessential amino } \\
\text { acids }\end{array}$ & Shaanxi, China & Hort16A & $\begin{array}{l}\text { Hitachi L-8900 amino } \\
\text { acid analyzer }\end{array}$ & Ripe fruits & $6.22 \mathrm{mg} / \mathrm{g} \mathrm{FW}$ & Ma et al., 2017 \\
\hline $\begin{array}{l}\text { Nonessential amino } \\
\text { acids }\end{array}$ & Shaanxi, China & Huayou & $\begin{array}{l}\text { Hitachi L-8900 amino } \\
\text { acid analyzer }\end{array}$ & Ripe fruits & $5.60 \mathrm{mg} / \mathrm{g} \mathrm{FW}$ & Ma et al., 2017 \\
\hline Total phenolic & New Zealand & $\begin{array}{l}\text { Zespri }^{\circledR} \text { SunGold } \\
\text { Kiwifruit }\end{array}$ & Folin-Ciocalteu method & $\begin{array}{l}\text { Thinned young } \\
\text { fruits ( } 20 \text { days) }\end{array}$ & 80 mg GAE/g FDW & Jiao et al., 2019 \\
\hline Total phenolic & Shanxi Province & Red sun & Folin-Ciocalteu method & Ripe fruits & $0.87 \mathrm{mg} \mathrm{GAE} / \mathrm{g} F W$ & Wang et al., 2018 \\
\hline Total phenolic & Shanxi Province & Cuiyu & Folin-Ciocalteu method & Ripe fruits & 0.83 mg GAE/g FW & Wang et al., 2018 \\
\hline Total flavonoid & New Zealand & $\begin{array}{l}\text { Zespri }^{\circledR} \text { SunGold } \\
\text { Kiwifruit }\end{array}$ & UVNis & $\begin{array}{l}\text { Thinned young } \\
\text { fruits (20days) }\end{array}$ & 30 mg CE/g FDW & Jiao et al., 2019 \\
\hline Total flavanol & New Zealand & $\begin{array}{l}\text { Zespri }^{\circledR} \text { SunGold } \\
\text { Kiwifruit }\end{array}$ & UVNis & $\begin{array}{l}\text { Thinned young } \\
\text { fruits (20days) }\end{array}$ & 20 mg CE/g FDW & Jiao et al., 2019 \\
\hline Total flavonoid & Shanxi Province & Red sun & UVNis & Ripe fruits & $0.68 \mathrm{mg} \mathrm{CE} / \mathrm{g} \mathrm{FW}$ & Wang et al., 2018 \\
\hline Total flavonoid & Shanxi Province & Cuiyu & UVNis & Ripe fruits & $0.68 \mathrm{mg} \mathrm{CE} / \mathrm{g} \mathrm{FW}$ & Wang et al., 2018 \\
\hline Total carotenoid & New Zealand & Hort16A & HPLC & $\begin{array}{l}\text { Main-harvested } \\
\text { fruits }\end{array}$ & $0.62 \mathrm{mg} / 100 \mathrm{~g} \mathrm{FW}$ & McGhie and Ainge, 2002 \\
\hline Total chlorophylls & New Zealand & gold kiwifruit & HPLC & Outer Pericarp & 0.07 mg/100 g FW & Montefiori et al., 2005 \\
\hline Total anthocyanins & New Zealand & Hongyang & HPLC & Pericarp & $2.99 \mathrm{mg} / 100 \mathrm{~g} \mathrm{FW}$ & Montefiori et al., 2005 \\
\hline Total organic acids & China & Hongyang & HPLC & Ripe Fruits & 39.86 mg/g FW & Montefiori et al., 2005 \\
\hline Total organic acids & China & Cuiyu & HPLC & Ripe Fruits & 29.65 mg/g FW & Montefiori et al., 2005 \\
\hline
\end{tabular}

Meanwhile, Jintao, Hort16A and Hongyang also had a high amount of nonessential amino acid and total free amino acid. The most abundant amino acids detected in the kiwifruit were arginine, glutamic acid, lysine, phenylalanine, aspartic acid, and tyrosine (Ma et al., 2017). There are a number of total saturated lipids including C8:0, C10:0, C12:0, C14:0, C16:0, and C18:0 with the content of $1.49,0.05,0.14,0.14,0.09,0.9$, and $0.14 \mathrm{mg} / \mathrm{g}$ in edible flesh portion of $A$. chinensis. Meanwhile, the content of monounsaturated fatty acids C16:1 and C18:1 are 0.09 and $0.27 \mathrm{mg} / \mathrm{g}$, and the polyunsaturated fatty acids C18:2 and C18:3 are 1.13 and $0.77 \mathrm{mg} / \mathrm{g}$ (Drummond, 2013). In fact, the kiwifruit seed oil is rich in unsaturated fatty acids (89.92\%), notably linolenic acid, which accounts for $60.59 \%$ of total seed oil (Luan et al., 2017). Ү-tocopherol, Y-tocotrienol, and $\mathrm{g}$-tocotrienol are identified in kiwifruit seed oil (Fiorentino et al., 2009). Besides, minerals like calcium, iron, potassium, magnesium, sodium, phosphorus, copper, manganese, zinc, iodine, selenium, and vitamins including vitamin A, $\beta$-carotene, lutein, zeaxanthin, riboflavin, niacin, pantothenic acid, vitamin B6, folate, tocopherol, vitamin $\mathrm{E}$, vitamin $\mathrm{K}$, and choline are identified in kiwifruit (Sivakumaran et al., 2018).
Thus, these data suggest that kiwifruit is an interesting fruit for daily nutrition and energy suppliers.

\section{Phytochemicals}

A range of phytochemicals, including triterpenoids, saponins, and phenolic compounds (flavonoids, polyphenols, anthraquinones, and coumarins) varying in structures, were found and identified in $A$. chinensis. The major constituents isolated and identified in leaves and roots of $A$. chinensis are listed in Table 4.

\section{Triterpenoids}

Currently, triterpenoids have been the major research focus of A. chinensis components due to their promising antitumor properties. To date, 42 triterpenoids have been isolated and identified mainly from roots of $A$. chinensis. The commonly triterpenoids found in roots of $A$. chinensis are 12-en-28-oic acids of oleanane and ursane type. It is noteworthy that some of these triterpenoids (1-2, 7, 15-18, 21, 25-26, 29-30, and 34-40) have significant antitumor activity and deserve further research and development. 
TABLE 4 | Chemical constituents isolated from $A$. chinensis.

\begin{tabular}{|c|c|c|c|c|c|}
\hline NO & Name & Cas & Formula & Source & Ref. \\
\hline \multicolumn{6}{|c|}{ Triterpenoids } \\
\hline 1. & $\begin{array}{l}(2 \alpha, 3 \beta, 4 \alpha)-2,3,23-T r i h y d r o x y u r s a-12,20(30) \text {-dien-28-oic acid; } \\
\text { Actinidic acid }\end{array}$ & $341971-45-7$ & $\mathrm{C}_{30} \mathrm{H}_{46} \mathrm{O}_{5}$ & $\begin{array}{l}\text { roots, } \\
\text { unripe fruit }\end{array}$ & $\begin{array}{l}\text { Ji and Liang, 1985; Lahlou } \\
\text { et al., } 2001\end{array}$ \\
\hline 2. & Maslinic acid & $4373-41-5$ & $\mathrm{C}_{30} \mathrm{H}_{48} \mathrm{O}_{4}$ & roots & Cui et al., 2007 \\
\hline 3. & Ursolic acid acetate & $7372-30-7$ & $\mathrm{C}_{32} \mathrm{H}_{50} \mathrm{O}_{4}$ & roots & Cui et al., 2007 \\
\hline 4. & 23-Hydroxyursolic acid & $94414-19-4$ & $\mathrm{C}_{30} \mathrm{H}_{48} \mathrm{O}_{4}$ & roots & Cui et al., 2007 \\
\hline 5. & Ergosta-4,6,8(14),22-tetraen-3-one & 19254-69-4 & $\mathrm{C}_{28} \mathrm{H}_{40} \mathrm{O}$ & roots & Cui et al., 2007 \\
\hline 6. & $2 \alpha, 3 \beta, 24$-Trihydroxyurs-12-en-28-oic acid & $143839-02-5$ & $\mathrm{C}_{30} \mathrm{H}_{48} \mathrm{O}_{5}$ & roots & Ji and Liang, 1985 \\
\hline 7. & $2 a, 3 a, 24$-Trihydroxyurs-12,20(30)-dien-28-oic acid & $341503-22-8$ & $\mathrm{C}_{30} \mathrm{H}_{46} \mathrm{O}_{5}$ & roots & Ji and Liang, 1985 \\
\hline 8. & Pygenic acid A (3-epi-corosolic acid) & $52213-27-1$ & $\mathrm{C}_{30} \mathrm{H}_{48} \mathrm{O}_{4}$ & roots & Chen et al., 2011 \\
\hline 9. & $2 \alpha, 3 \beta$-Dihydroxyurs-12-en-28,30-olide & $1198363-27-7$ & $\mathrm{C}_{30} \mathrm{H}_{46} \mathrm{O}_{4}$ & roots & Zhou et al., 2009 \\
\hline 10. & $2 \alpha, 3 \beta, 24$-Trihydroxyurs-12-en-28,30-olide & $1198363-28-8$ & $\mathrm{C}_{30} \mathrm{H}_{46} \mathrm{O}_{5}$ & roots & Zhou et al., 2009 \\
\hline 11. & 3ß-Hydroxyurs-12,18-dien-28-oic acid & $14021-14-8$ & $\mathrm{C}_{30} \mathrm{H}_{46} \mathrm{O}_{3}$ & roots & Zhou et al., 2009 \\
\hline 12. & 2a,3a,23-Trihydroxyursa-12, 20(30)-dien-28-oic acid & $1187824-97-0$ & $\mathrm{C}_{30} \mathrm{H}_{46} \mathrm{O}_{5}$ & roots & Zhou et al., 2009 \\
\hline 13. & $2 a, 3 \alpha, 19 a, 23,24-P e n t a h y d r o x y u r s-12-e n-28-o i c ~ a c i d$ & $1309360-33-5$ & $\mathrm{C}_{30} \mathrm{H}_{48} \mathrm{O}_{7}$ & roots & Xu et al., 2010 \\
\hline 14. & Ursolic acid & $74984-66-0$ & $\mathrm{C}_{30} \mathrm{H}_{48} \mathrm{O}_{3}$ & roots & Xu et al., 2010 \\
\hline 15. & Pseudotaraxasterol & $464-98-2$ & $\mathrm{C}_{30} \mathrm{H}_{50} \mathrm{O}$ & roots & Xu et al., 2010 \\
\hline 16. & 2a,3a,23-Trihydroxyurs-12-en-28-oic acid & $103974-74-9$ & $\mathrm{C}_{30} \mathrm{H}_{48} \mathrm{O}_{5}$ & roots & Xu et al., 2010 \\
\hline 17. & $2 \alpha, 3 \beta, 24$-Trihydroxyurs-12-en-28-oic acid & $475631-15-3$ & $\mathrm{C}_{30} \mathrm{H}_{48} \mathrm{O}_{5}$ & roots & Xu et al., 2010 \\
\hline 18. & $2 \alpha, 3 \beta, 19 \alpha, 23-T e t r a h y d r o x y u r s-12-e n-28-o i c$ acid & 70868-78-9 & $\mathrm{C}_{30} \mathrm{H}_{48} \mathrm{O}_{6}$ & roots & Xu et al., 2010 \\
\hline 19. & $\begin{array}{l}2 \alpha, 3 \alpha, 19 \alpha, 24-\text { Tetrahydroxyurs-12-en-28-oic acid } \\
28-O-\beta \text {-D-glucopyranoside }\end{array}$ & $153753-66-3$ & $\mathrm{C}_{36} \mathrm{H}_{58} \mathrm{O}_{11}$ & roots & Xu et al., 2010 \\
\hline 20. & Oleanolic acid acetate & $4339-72-4$ & $\mathrm{C}_{32} \mathrm{H}_{50} \mathrm{O}_{4}$ & roots & Zhu et al., 2013 \\
\hline 21. & Corosolic acid & $4547-24-4$ & $\mathrm{C}_{30} \mathrm{H}_{48} \mathrm{O}_{4}$ & roots & Zhu et al., 2013 \\
\hline 22. & Arjunic acid & $31298-06-3$ & $\mathrm{C}_{30} \mathrm{H}_{48} \mathrm{O}_{5}$ & roots & Zhu et al., 2013 \\
\hline 23. & Euscaphic acid & $53155-25-2$ & $\mathrm{C}_{30} \mathrm{H}_{48} \mathrm{O}_{5}$ & roots & Zhu et al., 2013 \\
\hline 24. & Oleanolic acid & $508-02-1$ & $\mathrm{C}_{30} \mathrm{H}_{48} \mathrm{O}_{3}$ & roots & He et al., 2014 \\
\hline 25. & 2a,3a,24-Trihydroxyolean-12-en-28-oic acid & $150821-16-2$ & $\mathrm{C}_{30} \mathrm{H}_{48} \mathrm{O}_{5}$ & roots & He et al., $2015 a$ \\
\hline 26. & $2 a, 3 a, 19 a, 24$-Tetrahydroxyurs-12-en-28-oic acid & $153753-65-2$ & $\mathrm{C}_{30} \mathrm{H}_{48} \mathrm{O}_{6}$ & roots & He et al., 2015a \\
\hline 27. & Jacoumaric acid & $63303-42-4$ & $\mathrm{C}_{39} \mathrm{H}_{54} \mathrm{O}_{6}$ & roots & Cui, 2016 \\
\hline 28. & $3 \beta$-Hydroxystigmast-5-en-7-one & $2034-74-4$ & $\mathrm{C}_{29} \mathrm{H}_{48} \mathrm{O}_{2}$ & roots & Xu et al., 2016 \\
\hline 29. & $\begin{array}{l}(2 a, 3 a)-2,3,23,24 \text {-Tetrahydroxyurs-12-en-28-oic acid; } 2 a, 3 a, 23 \text {, } \\
\text { 24-Tetrahydroxy ursan-12-en-28-acid }\end{array}$ & $143773-49-3$ & $\mathrm{C}_{30} \mathrm{H}_{48} \mathrm{O}_{6}$ & roots & Xu et al., 2016 \\
\hline 30. & $\begin{array}{l}\text { Oleanan-28-oic acid, 12-chloro-2,3,13,23-tetrahydroxy-, } \gamma^{-} \\
\text {lactone, }(2 \alpha, 3 \beta, 4 \alpha, 12 \alpha) \text { - }\end{array}$ & $1309360-32-4$ & $\mathrm{C}_{30} \mathrm{H}_{47} \mathrm{ClO}_{5}$ & roots & Xu et al., 2016 \\
\hline 31. & Urs-13(18)-en-28-oic acid, 2,3,23-trihydroxy-, $(2 \alpha, 3 \beta, 4 \alpha)$ - & $1980812-62-1$ & $\mathrm{C}_{30} \mathrm{H}_{48} \mathrm{O}_{5}$ & roots & Xu et al., 2016 \\
\hline 32. & $\begin{array}{l}\text { Urs-13(18)-en-28-oic acid, 2,3,19,23-tetrahydroxy-, } \beta \text {-D- } \\
\text { glucopyranosyl ester, }(2 \alpha, 3 \beta, 4 \alpha) \text { - }\end{array}$ & $1980812-63-2$ & $\mathrm{C}_{36} \mathrm{H}_{58} \mathrm{O}_{11}$ & roots & Xu et al., 2016 \\
\hline 33. & Pygenic acid B (2a,3a,24-trihydroxyurs-12-en-28-oic acid) & 89786-83-4 & $\mathrm{C}_{30} \mathrm{H}_{48} \mathrm{O}_{5}$ & roots & Xu et al., 2016 \\
\hline 34. & $2 a, 3 a, 23,24$-Tetrahydroxyursa-12, 20(30)-dien-28-oic acid & $2220160-45-0$ & $\mathrm{C}_{30} \mathrm{H}_{46} \mathrm{O}_{6}$ & roots & Wei et al., 2018 \\
\hline 35. & $2 \alpha, 3 \beta, 23,24$-Tetrahydroxyurs-12-en-28-oic acid & $116787-94-1$ & $\mathrm{C}_{30} \mathrm{H}_{48} \mathrm{O}_{6}$ & roots & Wei et al., 2018 \\
\hline 36. & $2 \alpha, 3 \beta, 23$-Trihydroxyurs-12-en-28-oic acid & $114580-55-1$ & $\mathrm{C}_{30} \mathrm{H}_{48} \mathrm{O}_{5}$ & roots & Wei et al., 2018 \\
\hline 37. & $3 \beta$-Hydroxyurs-12-en-28-oic acid & $77-52-1$ & $\mathrm{C}_{30} \mathrm{H}_{48} \mathrm{O}_{3}$ & roots & Wei et al., 2018 \\
\hline 38. & $3 \beta$-Hydroxyolean-12-en-28-oic acid & $28283-45-6$ & $\mathrm{C}_{35} \mathrm{H}_{56} \mathrm{O}_{7}$ & roots & Wei et al., 2018 \\
\hline 39. & $2 \beta, 3 \alpha, 23$-Trihydroxyurs-12-en-28-oic acid & $175132-32-8$ & $\mathrm{C}_{30} \mathrm{H}_{48} \mathrm{O}_{5}$ & roots & Wei et al., 2018 \\
\hline 40. & $2 \beta, 3 \beta, 23$-Trihydroxyurs-12-en-28-oic acid & $116348-15-3$ & $\mathrm{C}_{30} \mathrm{H}_{4} \mathrm{O}_{5}$ & roots & Wei et al., 2018 \\
\hline 41. & Spathodic acid 28-O- $\beta$-glucopyranoside & $870559-41-4$ & $\mathrm{C}_{36} \mathrm{H}_{58} \mathrm{O}_{10}$ & root barks & Zhang et al., 2018 \\
\hline 42. & Fupenzic acid & $119725-20-1$ & $\mathrm{C}_{30} \mathrm{H}_{44} \mathrm{O}_{5}$ & root barks & Zhang et al., 2018 \\
\hline \multicolumn{6}{|c|}{ Phenols } \\
\hline 43. & Planchol A & $883238-17-3$ & $\mathrm{C}_{14} \mathrm{H}_{14} \mathrm{O}_{6}$ & roots & Chang and Case, 2005 \\
\hline 44. & Planchol B & $883238-19-5$ & $\mathrm{C}_{15} \mathrm{H}_{16} \mathrm{O}_{6}$ & roots & Chang and Case, 2005 \\
\hline 45. & Planchol C & 883238-20-8 & $\mathrm{C}_{16} \mathrm{H}_{18} \mathrm{O}_{6}$ & roots & Chang and Case, 2005 \\
\hline 46. & Planchol D & $883238-21-9$ & $\mathrm{C}_{16} \mathrm{H}_{16} \mathrm{O}_{7}$ & roots & Chang and Case, 2005 \\
\hline 47. & $\begin{array}{l}\text { Benzeneacetic acid, 2-[(3,4-dihydroxybenzoyl)oxy]-4,6-dihydroxy- } \\
\text {, methyl ester }\end{array}$ & $911315-93-0$ & $\mathrm{C}_{16} \mathrm{H}_{14} \mathrm{O}_{8}$ & leaves & Wurms and Cooney, 2006 \\
\hline 48. & Tachioside (methoxyhydroquinone-3-O- $\beta$-D-glucopyranoside) & $109194-60-7$ & $\mathrm{C}_{13} \mathrm{H}_{18} \mathrm{O}_{8}$ & roots & Zhou et al., 2010 \\
\hline 49. & Isotachioside (methoxyhydroquinone-1-O- $\beta$-D-glucopyranoside) & $31427-08-4$ & $\mathrm{C}_{13} \mathrm{H}_{18} \mathrm{O}_{8}$ & roots & Zhou et al., 2010 \\
\hline 50. & Vanillic acid & $121-34-6$ & $\mathrm{C}_{8} \mathrm{H}_{8} \mathrm{O}_{4}$ & roots & Zhou et al., 2010 \\
\hline 51. & $\begin{array}{l}\text { 1-O-( } \beta \text {-D-glucosyl)-2-[2-methoxy-4-( } \omega \text {-hydroxypropyl)-phenoxy]- } \\
\text { propan-3-ol }\end{array}$ & $68340-35-2$ & $\mathrm{C}_{19} \mathrm{H}_{30} \mathrm{O}_{10}$ & roots & Zhou et al., 2010 \\
\hline 52. & Protocatechualdehyde & $139-85-5$ & $\mathrm{C}_{7} \mathrm{H}_{6} \mathrm{O}_{3}$ & roots & He et al., 2014 \\
\hline 53. & rel-(1R,2R)-1,2-Bis(4-hydroxy-3-methoxyphenyl)-1,3-propanediol & $69887-40-7$ & $\mathrm{C}_{17} \mathrm{H}_{20} \mathrm{O}_{6}$ & roots & He et al., 2014 \\
\hline 54. & rel-(1R,2S)-1,2-Bis(4-hydroxy-3-methoxyphenyl)-1,3-propanediol & $69887-41-8$ & $\mathrm{C}_{17} \mathrm{H}_{20} \mathrm{O}_{6}$ & roots & He et al., 2014 \\
\hline
\end{tabular}


TABLE 4 | Continued

\begin{tabular}{|c|c|c|c|c|c|}
\hline NO & Name & Cas & Formula & Source & Ref. \\
\hline 55. & p-Hydroxyl benzoic acid & $99-96-7$ & $\mathrm{C}_{7} \mathrm{H}_{6} \mathrm{O}_{3}$ & roots & He et al., 2014 \\
\hline 56. & Chlorogenic acid & $327-97-9$ & $\mathrm{C}_{16} \mathrm{H}_{18} \mathrm{O}_{9}$ & roots & He et al., $2015 a$ \\
\hline 57. & Caffeic acid & $331-39-5$ & $\mathrm{C}_{9} \mathrm{H}_{8} \mathrm{O}_{4}$ & roots & He et al., $2015 a$ \\
\hline 58. & Cryptochlorogenic acid & $905-99-7$ & $\mathrm{C}_{16} \mathrm{H}_{18} \mathrm{O}_{9}$ & roots & He et al., 2015a \\
\hline 59. & Neochlorogenic acid & $906-33-2$ & $\mathrm{C}_{16} \mathrm{H}_{18} \mathrm{O}_{9}$ & roots & He et al., $2015 a$ \\
\hline 60. & 5-O-Coumaroylquinic acid & $87099-71-6$ & $\mathrm{C}_{16} \mathrm{H}_{18} \mathrm{O}_{8}$ & roots & He et al., 2015a \\
\hline 61. & Dihydroxy-dihydrochalcone-2'-O- $\beta$-D-glucopyranoside & $23140-78-5$ & $\mathrm{C}_{21} \mathrm{H}_{24} \mathrm{O}_{9}$ & roots & Xu et al., 2016 \\
\hline \multicolumn{6}{|c|}{ Flavonoids } \\
\hline 62. & Epicatechin & $490-46-0$ & $\mathrm{C}_{15} \mathrm{H}_{14} \mathrm{O}_{6}$ & unknown & $\begin{array}{l}\text { Michaud and Ane-Margail, } \\
1977\end{array}$ \\
\hline 63. & epi-Afzelechin & $24808-04-6$ & $\mathrm{C}_{15} \mathrm{H}_{14} \mathrm{O}_{5}$ & unknown & $\begin{array}{l}\text { Michaud and Ane-Margail, } \\
1977\end{array}$ \\
\hline 64. & Procyanidin $\mathrm{C}_{1}$ & $37064-30-5$ & $\mathrm{C}_{45} \mathrm{H}_{38} \mathrm{O}_{18}$ & unknown & $\begin{array}{l}\text { Michaud and Ane-Margail, } \\
1977\end{array}$ \\
\hline 65. & $\begin{array}{l}\text { 2-(3,4-Dihydroxyphenyl)-3,4-dihydro-4-[(phenylmethyl)thio]-2H-1- } \\
\text { benzopyran-3,5,7-triol }\end{array}$ & $66052-27-5$ & $\mathrm{C}_{22} \mathrm{H}_{20} \mathrm{O}_{6} \mathrm{~S}$ & unknown & $\begin{array}{l}\text { Michaud and Ane-Margail, } \\
1977\end{array}$ \\
\hline 66. & $\begin{array}{l}\text { 2,2'-Bis(3,4-dihydroxyphenyl)-3,3',4,4'-tetrahydro-4'- } \\
\text { [(phenylmethyl)thio][4,8'-bi-2H-1-benzopyran]-3,3',5,5',7,7'-hexol }\end{array}$ & $66293-44-5$ & $\mathrm{C}_{37} \mathrm{H}_{32} \mathrm{O}_{12} \mathrm{~S}$ & unknown & $\begin{array}{l}\text { Michaud and Ane-Margail, } \\
1977\end{array}$ \\
\hline 67. & Afzelechin & $2545-00-8$ & $\mathrm{C}_{15} \mathrm{H}_{14} \mathrm{O}_{5}$ & roots & Chang and Case, 2005 \\
\hline 68. & Procyanidin $\mathrm{B}_{3}$ & 23567-23-9 & $\mathrm{C}_{30} \mathrm{H}_{26} \mathrm{O}_{12}$ & roots & Chang and Case, 2005 \\
\hline 69. & Procyanidol $\mathrm{B}_{2}$ & $29106-49-8$ & $\mathrm{C}_{30} \mathrm{H}_{26} \mathrm{O}_{12}$ & roots & Chang and Case, 2005 \\
\hline 70. & Afzelechin- $(4 a \rightarrow 8)$-afzelchin & $101339-37-1$ & $\mathrm{C}_{30} \mathrm{H}_{26} \mathrm{O}_{10}$ & roots & Chang and Case, 2005 \\
\hline 71. & $\begin{array}{l}\text { (2R,2'R,3R,3'R,4R)-3,3',4,4'-'Tetrahydro-2,2'-bis(4-hydroxyphenyl) } \\
\text { [4,8'-bi-2H-1-benzopyran]-3,3',5,5',7,7'-hexol }\end{array}$ & $114715-48-9$ & $\mathrm{C}_{30} \mathrm{H}_{26} \mathrm{O}_{10}$ & roots & Chang and Case, 2005 \\
\hline 72. & Quercetin & $117-39-5$ & $\mathrm{C}_{15} \mathrm{H}_{10} \mathrm{O}_{7}$ & fruits & Lee et al., 2010 \\
\hline 73. & $(+)$-Catechin & $154-23-4$ & $\mathrm{C}_{15} \mathrm{H}_{14} \mathrm{O}_{6}$ & roots & Zhou et al., 2010 \\
\hline 74. & (-)-Epicatechin-5-O- $\beta$-D-glucopyranoside & $131831-20-4$ & $\mathrm{C}_{21} \mathrm{H}_{24} \mathrm{O}_{11}$ & roots & He et al., 2014 \\
\hline \multicolumn{6}{|c|}{ Anthraquinones } \\
\hline 75. & Emodic acid & $478-45-5$ & $\mathrm{C}_{15} \mathrm{H}_{8} \mathrm{O}_{7}$ & roots & Ji and Liang, 1985 \\
\hline 76. & Hydroxyemodin & $481-73-2$ & $\mathrm{C}_{15} \mathrm{H}_{10} \mathrm{O}_{6}$ & roots & Ji and Liang, 1985 \\
\hline 77. & Emodin & $518-82-1$ & $\mathrm{C}_{15} \mathrm{H}_{10} \mathrm{O}_{5}$ & roots & Ji and Liang, 1985 \\
\hline 78. & Emodin 3-methyl ether & $521-61-9$ & $\mathrm{C}_{16} \mathrm{H}_{12} \mathrm{O}_{5}$ & roots & Ji and Liang, 1985 \\
\hline 79. & Questin & $3774-64-9$ & $\mathrm{C}_{16} \mathrm{H}_{12} \mathrm{O}_{5}$ & roots & Ji and Liang, 1985 \\
\hline \multicolumn{6}{|c|}{ Coumarins } \\
\hline 80. & 5-Hydroxy-6-methoxy-7-O- $\beta$-D-glucosyl coumarin & $141238-32-6$ & $\mathrm{C}_{16} \mathrm{H}_{18} \mathrm{O}_{10}$ & roots & Zhou et al., 2010 \\
\hline 81. & Fraxin & $524-30-1$ & $\mathrm{C}_{16} \mathrm{H}_{18} \mathrm{O}_{10}$ & roots & Zhou et al., 2010 \\
\hline 82. & Esculin & $531-75-9$ & $\mathrm{C}_{15} \mathrm{H}_{16} \mathrm{O}_{9}$ & roots & He et al., 2015b \\
\hline 83. & Isofraxoside & $24778-11-8$ & $\mathrm{C}_{16} \mathrm{H}_{18} \mathrm{O}_{10}$ & roots & He et al., 2015b \\
\hline \multicolumn{6}{|c|}{ Other compouds } \\
\hline 84. & $\beta$-Sitosterol & $83-46-5$ & $\mathrm{C}_{29} \mathrm{H}_{50} \mathrm{O}$ & roots & Ji and Liang, 1985 \\
\hline 85. & Butyl $\beta$-D-fructopyranoside & $67884-27-9$ & $\mathrm{C}_{10} \mathrm{H}_{20} \mathrm{O}_{6}$ & roots & Zhou et al., 2010 \\
\hline 86. & Lignoceric acid & $557-59-5$ & $\mathrm{C}_{24} \mathrm{H}_{48} \mathrm{O}_{2}$ & roots & Chen et al., 2011 \\
\hline 87. & (-)-Quinic acid $\gamma$-lactone & $665-27-0$ & $\mathrm{C}_{7} \mathrm{H}_{10} \mathrm{O}_{5}$ & roots & Chen et al., 2011 \\
\hline 88. & Stearyl- $\beta$-D-glucopyranoside & $76739-16-7$ & $\mathrm{C}_{24} \mathrm{H}_{48} \mathrm{O}_{6}$ & roots & Chen et al., 2011 \\
\hline 89. & Daucosterol & $474-58-8$ & $\mathrm{C}_{35} \mathrm{H}_{60} \mathrm{O}_{6}$ & roots & Chen et al., 2011 \\
\hline 90. & Indole-3-carboxylic acid & $771-50-6$ & $\mathrm{C}_{9} \mathrm{H}_{7} \mathrm{~N} \mathrm{O}_{2}$ & roots & He et al., 2014 \\
\hline 91. & Stigmastane-3,6-diol & $112244-29-8$ & $\mathrm{C}_{29} \mathrm{H}_{52} \mathrm{O}_{2}$ & roots & Cui, 2016 \\
\hline 92. & Sitoindoside I & 18749-71-8 & $\mathrm{C}_{51} \mathrm{H}_{90} \mathrm{O}_{7}$ & roots & Cui, 2016 \\
\hline
\end{tabular}

\section{Phenolic Compounds}

The phenolic compounds abundantly presented in different botanical parts of $A$. chinensis, and they have drawn increasing attention. These compounds include phenols, flavonoids, and flavanols are characterized by antitumor, antioxidant, and free radicals scavenging properties. HPLC-PAD and UPLC-QqQ-MS/ MS-based methods have been used generally for the identification and quantification of these phenolic compounds (Ma et al., 2017; Jiao et al., 2019). The total phenolic, flavonoid, and flavanol contents from young $A$. chinensis kiwifruits "Zespri ${ }^{\circledR}$ SunGold Kiwifruit" growing in 20 days are $82.84 \mathrm{mg}$ GAE/g FDW, 30.08 catechin/g equivalents FDW, and 20.20 catechin/g equivalents FDW. Meanwhile, the total phenolic, flavonoid, and flavanol contents presented in young $A$. chinensis kiwifruits growing in 60 days and mature kiwifruits are gradually decreasing, indicating polyphenol content possesses a decreasing pattern during fruit ripening (Jiao et al., 2019). The major chemical composition of phenolics detected in young "Zespri ${ }^{\circledR}$ SunGold Kiwifruit" are epicatechin, quercitrin, rutin, catechin, chlorogenic acid, ferulic acid, and vanillic acid. Based on UPLC-TOF/MS and UPLC-QqQ/ MS method, Zhao et al., 2014 showed that the radix A. chinensis contained catechin derivatives, quinic acid derivatives, coumarin 
derivatives, caffeic acid, and p-coumaric acid (Zhao et al., 2014), showing that $A$. chinensis appears to be a good source of phenolics.

\section{Volatile Compound and Essential Oil}

The volatile components of A. chinensis var. chinensis fruit and flowers have been profiled by GC-MS. The dominant volatile components of eating-ripe firmness fruit are straight-chain aldehydes, alcohols, and esters, such as hexanal, decanal, octanal, nonanal, benzaldehyde, acetaldehyde, hex-E2-enal, 1,8-cineole, ethanol, hexanol, methyl butanoate, and ethyl octanoate (Wang et al., 2011). The volatile components of flowers included (3E,6E)$\alpha$-farnesene (38.8\%), pentadecane (12.49\%), (+)-germacrene D (8.55\%), heptadecane (8.01\%), (8Z)-heptadecene $(7.72 \%)$, 2-phenylethano (4.69\%), (3Z,6Z,9Z)-heptadecatriene (2.54\%), and nonadecane (1.98\%) (Twidle et al., 2018). It can be found that terpenes and straight chain alkenes were dominant in flowers of A. chinensis var. chinensis, which contained nearly $>92 \%$ of the total ion counts. Importantly, many of these compounds possess strong and interesting aroma. However, the volatile components gradually changed during maturation. The essential oil of roots of A. chinensis have been profiled by GC-MS, and the major essential oil in roots are dodecane (29.39\%), octane $(5.16 \%)$, decane (2.94\%), paeonal (2.81\%), camphor (2.77\%), $n$-decanoic acid (2.64\%), 4-Methyldodecane (2.45\%), undecane (2.16\%), and linalool oxide (2.1\%) (Yu et al., 2009).

\section{Carotenoid and Chlorophyll}

Carotenoids and chlorophyll are responsible for the color and attractiveness of kiwifruit fruits, as well as provide nutritional values. The carotenoids detected in the red-fleshed genotypes of A. chinensis fruit (Hort16A) are 9'-cis-neoxanthin, violaxanthin, antheraxanthin, lutein, zeaxanthin, $\beta$-cryptoxanthin, and $\beta$-carotene (McGhie and Ainge, 2002; Montefiori et al., 2005). Chlorophylls $\mathrm{a}$ and $\mathrm{b}$ are the dominant chlorophylls in Hort16A (McGhie and Ainge, 2002).

\section{Quality Determination}

Ripe kiwifruit is susceptible to environmental and itself. Usually, human sensory evaluation method can directly identify the fruit shape, color, surface, pulp, and flavor, but there is little information about swelling, ripening, and other agents present in fruit. Physical and chemical method including firmness and microbial are used effectively to determine the quality condition of kiwifruit. Some new instrument detection methods with accurate analysis ability such as GC, GC-MS, HPLC, UPLC-QqQ-MS/MS, and electronic nose combined with surface acoustic wave resonator are developed for the fruit and its products quality rapid analysis (Kvesitadze et al., 2001; Montefiori et al., 2005; Liu and Hui, 2015; Jiao et al., 2019). As to radix A. chinensis, the systematical method like UPLC-TOF/MS and UPLC-QqQ/MS is commonly applied to quality evaluation and active components analysis for A. chinensis (Zhao et al., 2014). Therefore, there are many high accurate analysis methods for rapid quality evaluation, but there is a lack of effective and standardized quality and safety standard for kiwifruit in China. Thus, there is an urgent demand for developing specific functional components and quality evaluation indicators for standardization and quality control of the fruit and its products.

\section{Biological Activities}

A. chinensis contains a range of bioactive compounds accounting for natural pharmacological properties including antitumor, antioxidant, anti-inflammatory, immunoregulatory, hypolipemic, antidiabetic, and cardiovascular protective activities, and most of these biological activities support its traditional use. Table 5 shows the major biological activities of compound or extract from A. chinensis.

\section{Antitumor Activity}

Crude extracts, fractions, and isolated compounds from $A$. chinensis exhibited strong inhibition against tumor growth in various forms of human cancer cells. These cancer cells were hepatocellular carcinoma cells HepG2 (Xu et al., 2010; Zuo et al., 2012), Hep3B, SMMC7721, MHCC97L, MHCC97H, HCCLM3 (Fang et al., 2019), HL-7702 (He et al., 2017), Huh7 (Hou et al., 2018), lung cancer cells NCI-H460 and NCI-H1299 (Lv et al., 2018), colon cancer cells HT-29, LoVo, and SW480, pharyngeal carcinoma cell lines Fadu and HEP-2, gastric cancer cells SGC7901, BGC-823, MKN-49P, and MFC, as well as other cancer cells like A549, P-388, MCF-7, SK-OV-3, and HeLa (Chang and Case, 2005; Xu et al., 2010; Xu et al., 2010; Zuo et al., 2012; Shen et al., 2014; Xia et al., 2017; Gu et al., 2017; Wang et al., 2017; Wei et al., 2018). These reported antitumor activities are consistent with the traditional usage such as liver cancer, lung cancer, colon cancer, esophagus cancer, and gastric cancer.

A large number of triterpenoids in roots of $A$. chinensis especially those with carboxyl group showed marked cytotoxicity against various types of cancer cells in vitro. Especially, compounds 1-2, 7, 15-18, 21, 25-26, 29-30, 34-40, and 43-46 exhibited remarkable antitumor activity against on A549, HepG2, LVOV, MCF-7, HeLa, and/or HepG2 in vitro (Table 5). Additionally, the polysaccharide of Hogyang fruit showed notable inhibitory against tumor cells lines SGC7901, MCF7, HT29, HepG2, and NCI-H460 with $\mathrm{IC}_{50}$ of were $0.28,0.31$, $0.58,0.64$, and $0.65 \mu \mathrm{M}$, respectively (Xia et al., 2017). In vivo, a polysaccharide isolated from the roots of $A$. chinensis showed antitumor activity by prolonging the life of EAC or P388 cellsinduced tumor mice and inhibiting the DNA synthesis in EAC cells (Lin, 1988). Early treatment and long-term treatment with water extracts of roots from $A$. chinensis with $2 \mathrm{~g} / \mathrm{kg} /$ day strongly attenuated the malignant behavior of HCC in mice by decreasing DLX2 expression (Fang et al., 2019).

The molecular mechanism of the inhibition against tumor growth and the apoptosis promoting of the fractions and isolated compounds were due to downregulate $D L X 2$ gene expression and VEGFR2/Src/FAK pathway, inhibit cholesterol metabolism by upregulating PCSK9 signaling pathway, regulate gene encoding laminin subunit beta-3 pathways, and decreased NF- $\mathrm{KB}$ and EP3 expression. Meanwhile, the antioxidation and anti-inflammation are also important and possible mechanisms. The triterpenoids, polysaccharides, and phenolic compounds were identified as 
TABLE 5 | Biological activities of compounds or extracts of $A$. chinensis.

\begin{tabular}{|c|c|c|c|c|}
\hline Effect & Compound/Extract & $\begin{array}{l}\text { Class of } \\
\text { compounds }\end{array}$ & In vitro & Ref. \\
\hline \multirow[t]{18}{*}{ a } & 1 & A & $\begin{array}{l}\text { Showed cytotoxicities against HepG2, } \\
\text { A549, MCF-7, and SK-OV-3 with } \mathrm{IC}_{50} \\
(48 \mathrm{~h} \text { ) values of } 36.4,40.37,44.3 \text {, and } \\
16.33 \mu \mathrm{M} \text {. }\end{array}$ & Wei et al., 2018 \\
\hline & 2 & A & $\begin{array}{l}\text { Showed cytotoxicities against A549, } \\
\text { LoVo, and HepG2 with } \mathrm{IC}_{50}(48 \mathrm{~h}) \\
\text { values of } 23.2,6 \text {, and } 34.9 \mu \mathrm{g} / \mathrm{ml} \text {. }\end{array}$ & $\begin{array}{l}\text { Wei et al., 2018; Xu et } \\
\text { al., } 2010\end{array}$ \\
\hline & 7 & A & $\begin{array}{l}\text { Showed cytotoxicities against } A 549 \text {, } \\
\text { MCF-7, SK-OV-3, and HeLa with } I_{50} \\
(48 \text { h) values of } 16.63,47.93,22.91 \text {, } \\
\text { and } 15.27 \mu \mathrm{M} \text {. }\end{array}$ & Wei et al., 2018 \\
\hline & 15 & $A$ & $\begin{array}{l}\text { Showed cytotoxicities against LoVo, } \\
\text { and HepG2 with } \mathrm{IC}_{50}(48 \mathrm{~h}) \text { values of } \\
31.1 \text {, and } 33.9 \mu \mathrm{g} / \mathrm{ml} \text {. }\end{array}$ & Xu et al., 2010 \\
\hline & 16 & $A$ & $\begin{array}{l}\text { Showed cytotoxicities against HepG2, } \\
\text { MCF-7, SK-OV-3, and HeLa with } \mathrm{IC}_{50} \\
\text { ( } 48 \text { h) values of } 12.22,36.29,45.13 \text {, } \\
\text { and } 49.71 \mu \mathrm{M} \text {. }\end{array}$ & Wei et al., 2018 \\
\hline & 17 & $A$ & $\begin{array}{l}\text { Showed cytotoxicities against } A 549 \text {, } \\
\text { MCF-7, SK-OV-3, and HeLa with } I_{50} \\
(48 \text { h) values of } 39.3,11.01,40.9 \text { and } \\
41.6 \mu \mathrm{M} \text {. }\end{array}$ & Wei et al., 2018 \\
\hline & 18 & A & $\begin{array}{l}\text { Showed cytotoxicities against HepG2, } \\
\text { A549, MCF-7, and HeLa with } \mathrm{IC}_{50}(48 \\
\text { h) values of } 19.08,32.08,35.74 \text {, and } \\
15.05 \mu \mathrm{M} \text {. }\end{array}$ & Wei et al., 2018 \\
\hline & 21 & A & $\begin{array}{l}\text { Inhibited HCC cells migration by } \\
\text { targeting the VEGFR2/Src/FAK } \\
\text { pathway. }\end{array}$ & Ku et al., 2015 \\
\hline & 21 & A & $\begin{array}{l}\text { Showed cytotoxicities against A549, } \\
\text { LoVo, and HepG2 with } \mathrm{IC}_{50}(48 \mathrm{~h}) \\
\text { values of } 34.6,2.9 \text {, and } 9.2 \mu \mathrm{g} / \mathrm{ml} \text {. }\end{array}$ & Xu et al., 2010 \\
\hline & 25 & A & $\begin{array}{l}\text { Showed cytotoxicities against } \mathrm{A} 549 \\
\text { and SK-OV-3 with } \mathrm{IC}_{50}(48 \mathrm{~h}) \text { values of } \\
42.74 \text { and } 25.83 \mu \mathrm{M} \text {. }\end{array}$ & Wei et al., 2018 \\
\hline & 26 & A & $\begin{array}{l}\text { Showed cytotoxicities against A549 } \\
\text { and HeLa with } \mathrm{IC}_{50}(48 \mathrm{~h}) \text { values of } 22.6 \\
\text { and } 29.35 \mu \mathrm{M} \text {. }\end{array}$ & Wei et al., 2018 \\
\hline & 29 & $A$ & $\begin{array}{l}\text { Showed cytotoxicities against A549 } \\
\text { and SK-OV-3 with } \mathrm{IC}_{50}(48 \mathrm{~h}) \text { values of } \\
31.3 \text { and } 37.9 \mu \mathrm{M} \text {. }\end{array}$ & Wei et al., 2018 \\
\hline & 30 & $A$ & $\begin{array}{l}\text { Showed cytotoxicities against A549, } \\
\text { LoVo, and HepG2 with } \mathrm{IC}_{50}(48 \mathrm{~h}) \\
\text { values of } 30.4,31.1 \text {, and } 25.5 \mu \mathrm{g} / \mathrm{ml} \text {. }\end{array}$ & Xu et al., 2010 \\
\hline & 34 & $A$ & $\begin{array}{l}\text { Showed cytotoxicities against HepG2, } \\
\text { A549, MCF-7, and HeLa with } \mathrm{IC}_{50}(48 \\
\text { h) values of } 19.62,18.86,45.94 \text { and } \\
28.74 \mu \mathrm{M} \text {. }\end{array}$ & Wei et al., 2018 \\
\hline & 35 & A & $\begin{array}{l}\text { Showed cytotoxicities against HepG2, } \\
\text { MCF-7, and SK-OV-3 with } \mathrm{IC}_{50}(48 \mathrm{~h}) \\
\text { values of } 11.76,12 \text {, and } 10.3 \mu \mathrm{M} \text {. }\end{array}$ & Wei et al., 2018 \\
\hline & 36 & $A$ & $\begin{array}{l}\text { Showed cytotoxicities against HepG2, } \\
\text { MCF-7, and SK-OV-3 with } \mathrm{IC}_{50}(48 \mathrm{~h}) \\
\text { values of } 14.22,16.99,28.9 \mu \mathrm{M} \text {. }\end{array}$ & Wei et al., 2018 \\
\hline & 37 & A & $\begin{array}{l}\text { Showed cytotoxicities against HepG2, } \\
\text { A549, MCF-7, and SK-OV-3 with } \mathrm{IC}_{50} \\
(48 \text { h) values of } 48.4,12.7,11.2 \text {, and } \\
31.7 \mu \mathrm{M} \text {. }\end{array}$ & Wei et al., 2018 \\
\hline & 38 & $A$ & $\begin{array}{l}\text { Showed cytotoxicities against } A 549 \text {, } \\
\text { MCF-7, and SK-OV-3 with } \mathrm{IC}_{50}(48 \mathrm{~h}) \\
\text { values of } 34.45,42.2 \text { and } 49.55 \mu \mathrm{M} \text {. }\end{array}$ & Wei et al., 2018 \\
\hline
\end{tabular}


TABLE 5 | Continued

\begin{tabular}{|c|c|c|c|c|}
\hline Effect & Compound/Extract & $\begin{array}{l}\text { Class of } \\
\text { compounds }\end{array}$ & In vitro & Ref. \\
\hline & 39 & A & $\begin{array}{l}\text { Showed cytotoxicities against HepG2 } \\
\text { with } \mathrm{IC}_{50}(48 \mathrm{~h}) \text { values of } 32.5 \mu \mathrm{M} \text {. }\end{array}$ & Wei et al., 2018 \\
\hline & 40 & A & $\begin{array}{l}\text { Inhibited NCl-H460 cell proliferation by } \\
\text { decreasing NF-kB expression. Showed } \\
\text { cytotoxicities against SK-OV-3 with } \mathrm{IC}_{50} \\
\text { of } 37.21 \mu \mathrm{M} \text {. }\end{array}$ & $\begin{array}{l}\text { Cheng et al., 2015; Wei } \\
\text { et al., } 2018\end{array}$ \\
\hline & 43 & $\mathrm{~B}$ & $\begin{array}{l}\text { Showed cytotoxic activity against } \\
\text { P-388 and } A-549 \text { cell lines with } I_{50} \text { of } \\
2.5 \text { and } 1.42 \mu \mathrm{M} \text {. }\end{array}$ & Chang and Case, 2005 \\
\hline & 44 & $B$ & $\begin{array}{l}\text { Showed cytotoxic activity against } \\
\text { P-388 and } A-549 \text { cell lines with } I_{50} \text { of } \\
3.85 \text { and } 2.88 \mu \mathrm{M} \text {. }\end{array}$ & Chang and Case, 2005 \\
\hline & 45 & $\mathrm{~B}$ & $\begin{array}{l}\text { Showed cytotoxic activity against } \\
\text { P-388 and A-549 cell lines with } I_{50} \text { of } \\
5.02 \text { and } 4.5 \mu \mathrm{M} \text {. }\end{array}$ & Chang and Case, 2005 \\
\hline & 46 & $\mathrm{~B}$ & $\begin{array}{l}\text { Showed cytotoxic activity against } \\
\text { P-388 and A-549 cell lines with } I_{50} \text { of } \\
3.52 \text { and } 2.6 \mu \mathrm{M} \text {. }\end{array}$ & Chang and Case, 2005 \\
\hline \multirow[t]{11}{*}{$b$} & vitamin E ( $\mathrm{g}$-Tocomonoenol) & $\mathrm{C}$ & $\begin{array}{l}\text { Radical-scavenging capacities on } \\
\text { DPPH and } \mathrm{O}_{2} \text { were } 23.96 \text { and } 29.20 \% \text {; } \\
\text { hydroperoxide conjugate dienes } \\
\text { formation and TBARS were } 26.88 \text { and } \\
46.70 \% \text {. }\end{array}$ & Fiorentino et al., 2009 \\
\hline & vitamin E (a-tocopherol) & C & $\begin{array}{l}\text { Radical-scavenging capacities on } \\
\text { DPPH and } \mathrm{O}_{2} \text { were } 25.21 \text { and } 27.07 \% \text {. } \\
\text { hydroperoxide conjugate dienes } \\
\text { formation and TBARS were } 33.08 \text { and } \\
53.01 \% \text {. }\end{array}$ & Fiorentino et al., 2009 \\
\hline & vitamin E (g-tocopherol) & $\mathrm{C}$ & $\begin{array}{l}\text { Radical-scavenging capacities on } \\
\text { DPPH and } \mathrm{O}_{2} \text { were } 23.4 \text { and } 29.273 \% \text {; } \\
\text { hydroperoxide conjugate dienes } \\
\text { formation and TBARS were } 25.48 \text { and } \\
43.2 \% \text {. }\end{array}$ & Fiorentino et al., 2009 \\
\hline & $\begin{array}{l}\text { polymeric proanthocyanidins } \\
\text { fractionated by methanol- water } \\
(80: 20, v / v)\end{array}$ & $\mathrm{D}$ & $\begin{array}{l}\mathrm{IC}_{50} \text { for DPPH, ABTS were } 105.3 \text { and } \\
74.7 \mu \mathrm{g} / \mathrm{ml} \text {; FRAP values is } 7.4 \mathrm{mM} \\
\text { VCE } \mathrm{g} \text {. }\end{array}$ & Chai et al., 2014 \\
\hline & $\begin{array}{l}\text { polymeric proanthocyanidins } \\
\text { fractionated by acetone- } \\
\text { methanol-water }(40: 40: 20 \\
\text { v/v/v) }\end{array}$ & $\mathrm{D}$ & $\begin{array}{l}\mathrm{IC}_{50} \text { for DPPH, ABTS were } 67.7 \text { and } \\
60.1 \mu \mathrm{g} / \mathrm{ml} \text {; FRAP values is } 9.6 \mathrm{mM} \\
\text { VCE } \mathrm{g} \text {. }\end{array}$ & Chai et al., 2014 \\
\hline & $\begin{array}{l}\text { polymeric proanthocyanidins } \\
\text { fractionated by acetone-water } \\
(70: 30, v / v)\end{array}$ & $\mathrm{D}$ & $\begin{array}{l}\mathrm{IC}_{50} \text { for DPPH, ABTS were } 69.3 \text { and } \\
39.5 \mu \mathrm{g} / \mathrm{ml} \text {; FRAP values is } 9.6 \mathrm{mmol} \\
\text { VCE } \mathrm{g} \text {. }\end{array}$ & Chai et al., 2014 \\
\hline & $\begin{array}{l}\text { polyphenols compounds } \\
\text { ( } 55.10 \text { mg GAE/g DW), } \\
\text { contain p-hydroxybenzoic } \\
\text { acid, protocatechuic acid, and } \\
\text { p-coumaric acid. }\end{array}$ & $B$ & $\begin{array}{l}10-50 \mu \mathrm{g} / \mathrm{ml} \text { showed DPPH free radical } \\
\text { scavenging. }\end{array}$ & Deng et al., 2016 \\
\hline & $\begin{array}{l}\text { seed oil rich in unsaturated fatty } \\
\text { acid from Hongyang }\end{array}$ & $E$ & $\begin{array}{l}\mathrm{IC}_{50} \text { for DPPH, HO.scavenging capacity } \\
\text { were } 31.4 \text { and } 1.09 \text {; FRAP and ORAC } \\
\text { values were } 107.3 \mathrm{mg} \text { and } 1.09 \text { Trolox/ } \\
\mathrm{kg} .\end{array}$ & Deng et al., 2018 \\
\hline & $\begin{array}{l}\text { seed oil rich in unsaturated fatty } \\
\text { acid from Huayou }\end{array}$ & $E$ & $\begin{array}{l}\mathrm{IC}_{50} \text { for } \mathrm{DPPH}, \mathrm{HO} \cdot \text { scavenging capacity } \\
\text { were } 33.7 \text { and } 1.12 \text {; FRAP and ORAC } \\
\text { values were } 72.0 \mathrm{mg} \text { and } 1.72 \text { Trolox/ } \\
\mathrm{kg} \text {. }\end{array}$ & Deng et al., 2018 \\
\hline & $\begin{array}{l}\text { seed oil rich in unsaturated fatty } \\
\text { acid from Hort } 16 \mathrm{~A}\end{array}$ & $E$ & $\begin{array}{l}\mathrm{IC}_{50} \text { for } \mathrm{DPPH}, \mathrm{HO} \cdot \text { scavenging capacity } \\
\text { were } 32.4 \text { and } 1.04 \text {; FRAP and ORAC } \\
\text { values were } 3.3 \mathrm{mg} \text { and } 1.69 \text { Trolox/kg. }\end{array}$ & Deng et al., 2018 \\
\hline & water-soluble polysaccharides & $\mathrm{F}$ & $\begin{array}{l}0.5-3 \mathrm{mg} / \mathrm{ml} \text { showed DPPH radical } \\
\text { scavenging activity, protection of the } \\
\mathrm{HEK} 293 \text { cells from } \mathrm{H}_{2} \mathrm{O}_{2} \text { damage. }\end{array}$ & Zhang et al., 2015 \\
\hline
\end{tabular}


TABLE 5 | Continued

\begin{tabular}{|c|c|c|c|c|c|}
\hline Effect & Compound/Extract & $\begin{array}{l}\text { Class of } \\
\text { compounds }\end{array}$ & In vitro & In vivo & Ref. \\
\hline \multirow[t]{2}{*}{ c } & $\begin{array}{l}\text { polymeric proanthocyanidins } \\
\text { fractionated by methanol- water } \\
(80: 20, v / v)\end{array}$ & $\mathrm{D}$ & $\begin{array}{l}\text { Inhibited monophenolase and } \\
\text { diphenolase activity with } \mathrm{IC}_{50} \text { of } 180.2 \\
\text { and } 390.2 \mu \mathrm{g} / \mathrm{ml} \text {. }\end{array}$ & & Chai et al., 2014 \\
\hline & $\begin{array}{l}\text { polymeric proanthocyanidins } \\
\text { fractionated by acetone- } \\
\text { methanol- water }(40: 40: 20 \text {, } \\
v / v / v)\end{array}$ & $\mathrm{D}$ & $\begin{array}{l}\text { Inhibited monophenolase activity with } \\
1 \mathrm{I}_{50} \text { of } 80.1 \text { and } 192.6 \mu \mathrm{g} / \mathrm{ml} \text {. }\end{array}$ & & Chai et al., 2014 \\
\hline \multirow[t]{2}{*}{$d$} & $\begin{array}{l}\text { polyphenols compounds } \\
\text { (55.10 mg GAE/g DW), } \\
\text { contain p-hydroxybenzoic } \\
\text { acid, protocatechuic acid, } \\
\text { p-coumaric acid, etc. }\end{array}$ & B & $\begin{array}{l}20,40,60 \mu \mathrm{g} / \mathrm{ml} \text { for } 12 \mathrm{~h} \text { inhibit IL-1 } \beta \\
\text { and TNF- } \alpha \text { secretion in LPS-induced } \\
\text { RAW } 264.7 \text { cells. }\end{array}$ & & Deng et al., 2016 \\
\hline & water-soluble polysaccharides & $\mathrm{F}$ & $\begin{array}{l}50,100,200,300 \mu \mathrm{g} / \mathrm{ml} \text { reduce } \mathrm{NO} \\
\text { production of RAW } 264.7 \text { cells, and } \\
100,200 \text { and } 300 \mu \mathrm{g} / \mathrm{ml} \text { enhanced } \\
\text { phagocytic activity of RAW } 264.7 \text { cells. }\end{array}$ & & Zhang et al., 2015 \\
\hline e & seed oil rich in fatty acids & E & & $\begin{array}{l}1.0 \text { and } 3.0 \mathrm{~mL} / \mathrm{kg} / \text { day } \\
\text { for } 84 \text { days decreased } \\
\text { bodyweight and ameliorated } \\
\text { serum TC, TG, HDL-C, and } \\
\text { LDL-C levels in high-fat diet } \\
\text { treated mice. }\end{array}$ & Qu et al., 2019 \\
\hline$f$ & flavonoid-rich extract & G & $\begin{array}{l}\mathrm{IC}_{50} \text { of ACE inhibitory activity was } 12.81 \\
\mathrm{mg} / \mathrm{ml} \text {. }\end{array}$ & & Hettihewa et al., 2018 \\
\hline \multirow[t]{5}{*}{ j } & 21 & $A$ & $\begin{array}{l}50 \mu \mathrm{g} / \mathrm{ml} \text { showed inhibitory effects on } \\
\text { CYP2C19, CYP2D6, and CYP3A4 } \\
\text { with } 69.3,71.0 \text { and } 39.3 \text { of remaining } \\
\text { activity. }\end{array}$ & & Xu et al., 2016 \\
\hline & 25 & $A$ & $\begin{array}{l}10 \mu \mathrm{g} / \mathrm{ml} \text { showed inhibitory effects on } \\
\text { CYP2C9, CYP2C19, CYP2D6, and } \\
\text { CYP3A4 with 28.3, 59.9, 31.8, and } \\
37.1 \% \text { of remaining activity. }\end{array}$ & & Xu et al., 2016 \\
\hline & 30 & A & $\begin{array}{l}10 \mu \mathrm{g} / \mathrm{ml} \text { showed inhibitory effects on } \\
\text { CYP2C9 and CYP3A4 with } 67.1 \text { and } \\
\text { 9.8\% of remaining activity. }\end{array}$ & & Xu et al., 2016 \\
\hline & 33 & A & $\begin{array}{l}50 \mu \mathrm{g} / \mathrm{ml} \text { showed inhibitory effects on } \\
\text { CYP2C19 and CYP3A4 with } 75.0 \text { and } \\
35.0 \text { of remaining activity }\end{array}$ & & Xu et al., 2016 \\
\hline & 61 & B & $\begin{array}{l}10 \mu \mathrm{g} / \mathrm{ml} \text { showed inhibitory effects } \\
\text { on CYP2C9 with } 69.0 \% \text { of remaining } \\
\text { activity. }\end{array}$ & & Xu et al., 2016 \\
\hline
\end{tabular}

a, Antitumor effects; b, Antioxidant activity; c. Antityrosinase activity; d, Anti-inflammatory activity; e, Hypolipidemic activity; $f$, ACE inhibitory activity;

g. Digestive activity; $h$, Antifungal activity; i, Antiviral activity; j, Cytochrome P450 enzyme inhibitory activity. A, Triterpenoid; B, Phenols; C, Vitamin; D, Proanthocyanidins; E, Oil; F, Polysaccharides; G, Flavonoids; H, Protein. 
the major bioactive compounds in the extract from $A$. chinensis roots with antitumor properties (Chang and Case, 2005; Wei et al., 2018), which provides new way to search for treating cancers with natural therapeutic compounds. Overall, A. chinensis has prominent antitumor potential and has a good health benefit for people, however, the further in vivo and clinical studies on antitumor properties of $A$. chinensis are needed for confirmation.

\section{Antioxidant Activity}

Antioxidant activity of bioactive compounds of $A$. chinensis have been the mostly studied by various in vitro and in vivo assays. These in vitro assays consisted of both chemical and biological assays like DPPH, ABTS, FRAP, HO, ORAC, oxidative stress by $\mathrm{H}_{2} \mathrm{O}_{2}$, and lipid oxidation (Chai et al., 2014; Lee et al., 2015; Hwang et al., 2017; Deng et al., 2018). The in vivo assays were based on SOD, GSH, ALT, AST, oxidative DNA damage, and lipid oxidation (Iwasawa et al., 2011; Sun et al., 2017; Deng et al., 2018; Wang et al., 2018). The above results showed that $A$. chinensis is a good source of bioactive compounds with antioxidant properties to various extents. The antioxidant capacities of kiwifruit are greatly attributed to polyphenols, flavonoid, unsaturated fatty acid, and vitamin C. In addition, the different extraction methods, different plant parts, and genetic diversity of kiwifruit demonstrated different antioxidant activities. The peel showed the strongest antioxidant activity, followed by the pulp and the core. The antioxidant activity of kiwifruit peel was mainly depended on plenty of phenolic substances, and the antioxidant activity of the pulp was mainly attributed to the existence of a large amount of vitamin C (Zhang et al., 2016). The seed oil of Hort 16A and Hongyang are attractive materials rich in unsaturated fatty acid demonstrated radical scavenging capacities for FRAP, DPPH, HO', and ORAC with $\mathrm{IC}_{50}$ of 3.3 mgTrolox/kg, $32.4 \mathrm{mg} / \mathrm{ml}, 1.04 \mathrm{mg} / \mathrm{ml}, 1.69 \mathrm{mgTrolox} / \mathrm{kg}$, and $107.3 \mathrm{mgTrolox} / \mathrm{kg}, 31.4 \mathrm{mg} / \mathrm{ml}, 1.09 \mathrm{mg} / \mathrm{ml}, 1.99 \mathrm{mgTrolox} / \mathrm{kg}$, respectively (Deng et al., 2018). The radical scavenging capacities of fresh and freeze-dried Hort 16A rich in phenolics and flavonoids for ABTS, DPPH, and ORAC were 8.8, 8.8, 98.3, and 6.0, 5.0, and $40.3 \mathrm{mg} \mathrm{VCE} / \mathrm{g}$, respectively (Hwang et al., 2017). The radical scavenging capacities of Red sun and Cuiyu rich in phenolics and flavonoids for ABTS, DPPH, ORAC, and FRAP were $1.35,1.01,10.78,1.50$ and $1.32,0.9,8.87,1.28 \mathrm{mg} \mathrm{VCE} / \mathrm{g}$, respectively (Wang et al., 2018). Oral administration of kiwifruit protected lymphocytes against oxidative DNA damage, inhibit lipid oxidation in mice, increased SOD and GSH, and lowered ALT and AST levels in the patients (Sun et al., 2017). Therefore, A. chinensis possess confirmed antioxidant capacity and it seems that appropriate extraction methods, appropriate genotypes, and plant parts can be screened to maximize the antioxidant properties of $A$. chinensis.

\section{Anti-Inflammatory Activity}

Anti-inflammatory activity of $A$. chinensis has been proved in vivo and in vitro models. On high-fat diet-induced obese C57BL/6 mice models, consecutive consumption the seeds oil of A. chinensis with 1.0 and $3.0 \mathrm{ml} / \mathrm{kg} \cdot \mathrm{bw}$ ameliorated obesity-induced inflammation by down-regulating the mRNA expression of related to inflammation adipokines, such as TNF- $\alpha$, IL-6, IL-1 $\beta$, COX-2, and iNOS (Qu et al., 2019). The aqueous and ethyl acetate extracts demonstrated anti-inflammatory activity in inflammatory bowel disease models of the $I L-10$ gene-deficient mice (Edmunds et al., 2012). In patients with type-2 diabetes mellitus, the fruit juice of $A$. chinensis showed preventative activity on inflammation by activating Keap1 and Nrf2 via upregulating miR-424 (Sun et al., 2017). On the cellular level, polyphenols mainly composed of protocatechuic acid, $p$-hydroxybenzoic acid, $p$-coumaric acid, caffeic acid, and ferulic acid from seeds of $A$. chinensis at concentration of 40 and $60 \mu \mathrm{g} / \mathrm{ml}$ for $12 \mathrm{~h}$ decreased the secretion of pro-inflammatory cytokines IL- $1 \beta$ and TNF- $\alpha$ in LPS-induced RAW 264.7 cells (Deng et al., 2016). Therefore, the anti-inflammatory potential $A$. chinensis seeds mainly depend on the synergetic effect of these polyphenols, and it may be used to prevent a variety of inflammation related diseases.

\section{Antibacterial Activity}

All the extracts including skin, pulp, seeds, and stems showed bactericidal against Staphylococcus aureus, Streptococcus pyogenes, S. faecalis, Salmonella typhi, Proteus mirabilis, Pseudomonas aeruginosa, Escherichia coli, and Klebsiella pneumonia. The skin and pulp extracts showed inhibition activity against S. aureus and S. pyogenes with MIC values of 8 and $4 \mu \mathrm{g} /$ $\mathrm{ml}$, but they showed moderate inhibition activity against $\mathrm{S}$. faecalis, S. typhi, P. mirabilis, P. aeruginosa, E. coli, and K. pneumonia with MIC values ranging from 16 to $128 \mu \mathrm{g} / \mathrm{ml}$. The leaves and stems extract just inhibited S. pyogenes and P. aeruginosa with MIC values of both 64 and $32 \mu \mathrm{g} / \mathrm{ml}$. The seeds extracts showed an exclusively bacteriostatic activity against these selected strains of bacteria with MIC values of between 1 and $8 \mu \mathrm{g} / \mathrm{ml}$ (Basile et al., 1997). Polyphenol from seeds of A. chinensis showed significant bactericidal against Bacillus cereus, B. subtilis, Shigella flexneri, and Salmonella Typhi, and bacteriostatic against B. thuringiensis. We can find that the antimicrobial activity of the polyphenol extract on gram-positive bacteria is higher than that of gramnegative bacteria (Deng et al., 2013). Therefore, kiwifruit seeds are potential food processing material for their antimicrobial activity.

\section{Immunoregulatory Activity}

Consumption of the aqueous extracts of whole fresh fruit of Hort16Aat $375 \mathrm{mg} / \mathrm{kg}$ for 12 days enhanced both innate and acquired immunity in cholera vaccine and tetanus/diphtheria vaccine models in Balb/c mice, showing a beneficial effect on healthy (Shu et al., 2008). The homopolysaccharide derivatived by $\mathrm{O}$-sulfation from the roots of $A$. chinensis at concentration of 10 and $50 \mu \mathrm{g} / \mathrm{ml}$ activated phagocytic activity and increased NO production of RAW 264.7 macrophages, and the activity of sulfated polysaccharides is strongly related to the degree of the sulfation (Niu et al., 2016), and treatment with $50-300 \mu \mathrm{g} / \mathrm{ml}$ water-soluble polysaccharides dose-dependently stimulated NO production and phagocytic activity of RAW 264.7macrophages (Zhang et al., 2015). It remains to clarify the detailed mechanism of immunoregulatory activity and the responsible compositions for this valid action. 


\section{Hypolipemic and Antidiabetic Activities}

Administered the seed oil of $A$. chinensis rich in fatty acids at 1.0 and $3.0 \mathrm{ml} / \mathrm{kg} \cdot \mathrm{bw}$ daily over 12 consecutive weeks significantly lowered bodyweight gain, inguinal fat tissue weight, and the accumulation of TC, TG, HDL-C, and LDL-Cin liver of thehigh-fat diet-induced obese C57BL/6 mice. Meanwhile, long-term consumption of the seed oil of $A$. chinensis up-regulated the expression of thermogenesis-related genes like PPAR- $\gamma, U C P 1, P G C 1-\alpha$, and PRDM16, down-regulated FAS expression, and altered the gut microbiota by decreasing the Firmicutes-to-Bacteroidetes ratio (Qu et al., 2019). In addition, the seed oil from $A$. chinensis supplementation improved insulin resistance and alleviated hyperglycemia by reducing HOMA-IR index and blood glucose in high fat diet-induced obese mice $(\mathrm{Qu}$ et al., 2019). Thus, the lipid lowering potential of $A$. chinensis seed provide a basis theory for food industries.

\section{Cardiovascular Protective Effects}

In $\mathrm{H} 9 \mathrm{c} 2$ rat cardiac myocytes cells induced by hypoxia in cardiomyocytes treated with angiotensin II, treatment with 1.25 and $2.5 \mathrm{mg} / \mathrm{ml}$ polysaccharide of $A$. chinensis alleviated cardiac hypertrophy, decreased mitochondrial dysfunction and reduced cardiomyocytes apoptosis by decreasing the apoptosisassociated genes expression like mitochondria associated 1 and caspases $3 / 8 / 9$, and cleaving caspases-3/8/9. Additionally, the protective effects of polysaccharide against hypoxia-induced apoptosis may be attributable to inactivate the ERK1/2 and PI3K/ AKT signaling pathways (Wang et al., 2018). The polysaccharide of $A$. chinensis can be potentially used in the treatment of heart disease. However, it is noteworthy that polysaccharide at high dose $(10 \mathrm{mg} / \mathrm{ml})$ suppressed the cardiomyocytes viability.

\section{Hypnotic Effects}

Oral administration of ethanol extracts from A. chinensis peel at dose of 250,500, and 1,000 $\mathrm{mg} / \mathrm{kg}$ dose-dependently decreased sleep latency and increased sleep duration in pentobarbitaltreated mice. Especially, the sequentially partitioned with ethyl acetate fraction rich in flavonoids $(1.63 \mathrm{mg} \mathrm{QE} / \mathrm{g})$ at $250 \mathrm{mg} / \mathrm{kg}$ exert significantly hypnotic effects and this sedative-hypnotic activity could be inhibited by $\mathrm{GABA}_{\mathrm{A}}$-BZD receptor antagonist flumazenil. The flavonoids may be attributable to hypnotic activity via allosteric $\mathrm{GABA}_{\mathrm{A}}$-BZD receptor modulation, but the precise mechanisms and the existing individual flavonoids are needed to be evaluated in the future (Yang et al., 2013).

\section{Ace Inhibitory Activity}

The $70 \%$ aqueous acetone extracts partitioning with hexane rich in flavonoid from Hort 16A dose-dependently inhibited ACE activity with $\mathrm{IC}_{50}$ of $12.81 \mathrm{mg} / \mathrm{ml}$ using a fluorescence-based biochemical assay. LC-MS/MS showed that the higher total phenolic and total flavonoid contents are identified in this extract. UPLC-MS/MS showed that polyphenols $(231.32 \mu \mathrm{g} / \mathrm{g}$ DW $)$ in the extract are mainly flavonols, flavanols, and phenolic acids. Specifically, quercetin-3-Ogalactoside $(205.19 \mu \mathrm{g} / \mathrm{g} \mathrm{DW})$, quercetin-3-O-glucoside $(0.45 \mu \mathrm{g} / \mathrm{g}$ DW), quercetin-3-O-rhamnoside $(0.61 \mu \mathrm{g} / \mathrm{g} \mathrm{DW})$, quercetin-3-Orutinoside $(0.29 \mu \mathrm{g} / \mathrm{g} \mathrm{DW})$, epicatechin $(5.15 \mu \mathrm{g} / \mathrm{g} \mathrm{DW})$, catechin
$(0.75 \mu \mathrm{g} / \mathrm{g} \mathrm{DW})$, epigallocatechin $(0.61 \mu \mathrm{g} / \mathrm{g} \mathrm{DW})$, phloridzin $(2.03$ $\mu \mathrm{g} / \mathrm{gDW})$, and isoferulic acid (15.12 $\mu \mathrm{g} / \mathrm{g} \mathrm{DW})$ are major compounds in the extract (Hettihewa et al., 2018). These compounds could be responsible for the observed in vitro ACE inhibitory activity of Hort $16 \mathrm{~A}$ fruit, though the active compounds identifying and in vivo animal studies remain to be investigated and conducted.

\section{Dermatological Activity}

The raw polysaccharides with $>90 \%$ carbohydrate and $5.2 \%$ residual protein from the fresh fruit of $A$. chinensis at $10 \mu \mathrm{g} / \mathrm{ml}$ showed a significantly proliferation-promoting on cell proliferation rates of HaCaT cell line and primary keratinocytes (NHK), and it also significantly promoted proliferation of human dermal fibroblasts at 132 and $198 \mu \mathrm{g} / \mathrm{ml}$. Meanwhile, treatment of the polysaccharides at $200 \mu \mathrm{g} / \mathrm{ml}$ significantly stimulated ATP-synthesis, promoted mitochondrial activity and energy metabolism of HaCaT keratinocytes, and significantly increased collagen synthesis in dermal skin equivalents (Deters et al., 2005). Kiwifruit pericarp proanthocyanidins mainly contained B-type propelargonidins, procyanidins, procyanidins gallate, and prodelphinidins showed strongly inhibition activity on tyrosinase, indicating that it can be used as whitening agents (Chai et al., 2014).

\section{Cytochrome P450 Enzyme Inhibitory Activities}

Cytochrome P450 system in liver plays an important role in drug metabolism. It transforms drug from hydrophobic to hydrophilic, which is easier to excrete. The $90 \% \mathrm{EtOH}$ extract of $A$. chinensis root at $50 \mu \mathrm{g} / \mathrm{ml}$ exhibited inhibition activities on CYP2C9, CYP2D6, and CYP3A4 in human liver tissue with the 69.0, 76.3, and 53.3\% of remaining activity, respectively. The inhibitory effect of the crude extract could be largely attributed to the presence of triterpenoids (Xu et al., 2016). It is worth noting that the combination of crude extracts or these triterpenoids with other medical herbs or drugs may lead to drug interaction with cytochrome CYPs at pharmacokinetic and pharmacodynamic levels, which indicates that people should cautiously consume $A$. chinensis fruit when taken medicine.

\section{Processing and Utilization}

Chinese kiwifruit is a very high nutritional value of nourishing and consumers' favorite fruit, which has shown application potential in food, medicine, and health products industry. China is the largest kiwifruit producer in the world. In 2016, kiwifruit production in China reached 2.41 million tons per year, accounting for $56.0 \%$ of the world's total kiwifruit production (United Nations Food and Agriculture Organization, 2016). To date, a series of commercially available products has been processed due to abundant nutrient substance and claimed health benefits. These Chinese kiwifruit related products include sliced fruit, juice, preserved fruit, yogurt, wine, canned fruit, dried kiwi slices, fruit vegetable juice drinks, biscuits, milk beverage, whipped cream, baked goods, vinegar, and oil capsule. Furthermore, various different parts of $A$. chinensis showed different uses. Briefly, the leaves contain protein, starch, and polyphenols, which may be developed as an excellent source of natural products. The beautiful and fragrant of Chinese 
kiwifruit flowers rich in honey juice and volatiles can be used as high-quality honey source. Kiwifruit peel residue as sources of high-quality pectin can be used as functional ingredient for food products. Chinese kiwifruit seeds rich in essential fatty acids, protein, and dietary fiber can be used in food and health products industry (Xie, 1975; Garcia et al., 2012). The roots and barks contain ursolic acid, oleanolic acid, and quercetin, which have antitumor effect against liver cancer, lung cancer, gastric cancer, esophageal cancer, colorectal cancer, and cervical cancer (Chang and Case, 2005; Xu et al., 2010; Wei et al., 2018). The different parts of $A$. chinensis are widely used as pharmaceutical raw materials in medicine for prevention and treatment of tumors. In addition, the various claimed nutritional and pharmacological properties including strong antitumor, antioxidation, and antiinflammatory potential of various extracts or active compounds of $A$. chinensis indicated that they could be further developed for functional food with added-commercial value or effective and safe drug formulations.

\section{Storage Methods}

Chinese kiwifruit has a short postharvest life because of fast softening and serious decay. Preservation of Chinese kiwifruit for prolonged periods is particularly important. Freezing and frozen storage is currently the most common method, which can effectively inhibit the softening of kiwifruit and prolong its postharvest life. However, kiwifruit is cold-sensitive and very susceptible to chilling injury when storage at the temperature between $-2^{\circ} \mathrm{C}$ and $2.5^{\circ} \mathrm{C}$ for a long time (Gerasopoulos et al., 2006; Ma et al., 2014). Interestingly, dipped by water for $10 \mathrm{~min}$ at $45^{\circ} \mathrm{C}$ to low temperature storage can prevent chilling injury development to kiwifruit. Meanwhile, the kiwifruit pretreated at $45^{\circ} \mathrm{C}$ and then stored at $0^{\circ} \mathrm{C}$ for 90 days showed higher firmness and soluble solids content, and MDA content and lipoxygenase activity in kiwifruit are reduced. However, pretreated at 20 and $55^{\circ} \mathrm{C}$ were ineffective at alleviating chilling tolerance (Ma et al., 2014). Various other treatments including preharvest calcium chloride sprays (Gerasopoulos and Drogoudi, 2005), putrescine (Yang et al., 2016), preharvest chilling (Sfakiotakis et al., 2005), and gradual cooling (Yang et al., 2013) have also been used to alleviate chilling injury in kiwifruit.

After harvest, kiwifruit is highly perishable, and its nutritional ingredients and quality decline rapidly due to the influence of internal biochemical reactions and external environment. The modified atmosphere packaging, chitosan, 1-methylcyclopropene, $\mathrm{ClO}_{2}$, ozone, tea polyphenols, protein, lipid composite film, oxalate, salicylic acid, and citric acid have been used individually or combined to alleviate physicochemical quality changes for postharvest of kiwifruit (Huang et al., 2017). The ozone treatment induced the ripening process, delayed the microbial growth, and influenced the content of vitamin C, polyphenols, flavonoids, and carotenoids (Goffi et al., 2019). The chitosan combined with salicylic acid treatment during storage at room temperature for 14 days provides a significantly effective preservative effect by delayed vitamin $\mathrm{C}$ and soluble solids decomposition, inhibiting moisture loss and acidity change, and maintaining texture and surface color of Chinese kiwifruit in 14 days of storage at room temperature (Huang et al., 2017).

\section{CONCLUSIONS}

Chinese kiwifruit and related products are increasingly popular throughout the world due to the remarkably economic, nutritional, and health benefits values. It is a good source of phenolic compounds, vitamin $\mathrm{C}$, carbohydrates, sugars, amino acids, and minerals. Of particular note in kiwifruit is vitamin $\mathrm{C}$ and minerals K. The phenolic compounds present in Chinese kiwifruit are organic acids and flavonoids, and fruit peel and flesh, leaf, vine, and roots also contain a variety of these phenolic components. The major components of the roots are triterpenoids characterized by 12 -en-28-oic acids of oleanane and ursane type. Terpenes, straight chain alkenes, alcohols, and esters were dominant volatile components in flowers and roots of $A$. chinensis. These chemical compounds render the $A$. chinensis with a range of sensory quality, nutritional, and pharmacological properties as proved by in vitro and in vivo studies. The claimed biological activity of isolated compounds, fractions, or crude extracts include antitumor, antioxidant, anti-inflammatory, antibacterial, immunoregulatory, hypolipemic, antidiabetic, and cardiovascular protective effects. Of particular note is that these claimed biological activities such as antitumor, antioxidant, and immunoregulatory may be greatly attributed to the existence of triterpenoids, polyphenols, flavonoid, polysaccharide, unsaturated fatty acid, and vitamin C. These findings suggest that Chinese kiwifruit can be useful in the prevention and treatment of pathologies associated to cancer, oxidative stress, and aging.

There are also research opportunities to better development, utilization, and protection kiwifruit for human consumption. Cytochrome P450 inhibitory activities, toxicity analysis, qualitative and quantitative metabolite research, effective and standardized quality standard building, and clinical studies should be encouraged to conducted for safe daily consumption. Meanwhile, the synergism and attenuation effects, metabolic behavior of various ingredients, as well as the in vivo and molecular mechanisms studies responsible for the observed biological properties should be conducted. It is also found that some of the $A$. chinensis cultivars were only supported by a few studies, and confirmative studies should be conducted to verify their health effects. Apart from the fruit, other plant parts of kiwifruit including leaves and roots should also be explored for effective utilization. The effective method and technology for the storage and preservation of kiwifruit during preharvest and postharvest remain to be explored to avoid the frequent chilling damage, soft rot, and mildew, and also decrease and improve the change of the chemical profile and bioactivity properties during storage.

\section{AUTHOR CONTRIBUTIONS}

$\mathrm{XC}$ and YM obtained the literatures. JF, ZZ, and XH wrote the manuscript. XH, LH, and YL gave ideas and edited the manuscript. All authors approved the paper for publication. 


\section{REFERENCES}

Basile, A., Vuotto, M. L., Violante, U., Sorbo, S., Martone, G., and CastaldoCobianchi, R. (1997). Antibacterial activity in Actinidia chinensis, Feijoa sellowiana and Aberia caffra. Inter. J. Antimicrob. Ag. 8, 199-203. doi: 10.1016/ S0924-8579(97)00376-2

Chai, W. M., Shi, Y., Feng, H. L., Xu, L., Xiang, Z. H., Gao, Y. S., et al. (2014). Structure characterization and antityrosinase mechanism of polymeric proanthocyanidins fractionated from kiwifruit pericarp. J. Agric. Food Chem. 62, 6382-6389. doi: 10.1021/jf501009v

Chang, J., and Case, R. (2005). Cytotoxic phenolic constituents from the root of Actinidia chinensis. Planta Med. 71, 955-959. doi: 10.1055/s-2005-871225

Chen, X., Yang, S., and Bai, S. (2011). Chemical constituents in root of Actinidia chinensis. Zhongcaoyao. 42, 841-843. doi: 10.7666/d.y1883489

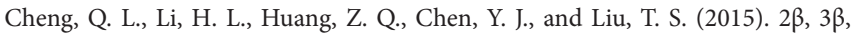
23-trihydroxy-urs-12-ene-28-olic acid (TUA) isolated from Actinidia chinensis radix inhibits NCI-H460 cell proliferation by decreasing NF- $\mathrm{\kappa B}$ expression. Chem.Biol. Interact. 240, 1-11. doi: 10.1016/j.cbi.2015.06.038

Cui, Y. (2016). Study on chemical constituents in ethyl acetate extracts from the root of Actinidia chinensis. Hubei Agri. Sci. 55, 5359-5360. doi: 10.14088/j.cnki. issn0439-8114.2016.20.048.

Cui, Y., Zhang, X., Chen, J., Zhang, Y., Lin, X., and Zhou, L. (2007). Chemical constituents from root of Actinidia chinensis. Zhongguo Zhongyao Zazhi. 32, 1663-1665. doi: 10.3321/j.issn:1001-5302.2007.16.016

Deng, J., Liu, Q., Zhang, C., Cao, W., Fan, D., and Yang, H. (2016). Extraction optimization of polyphenols from waste kiwi fruit seeds (Actinidia chinensis Planch.) and evaluation of its antioxidant and anti-inflammatory properties. Molecules 21, pii: E832. doi: 10.3390/molecules21070832

Deng, J. J., Liu, Q. Q., Zhang, Q., Zhang, C., Liu, D., Fan, D. D., et al. (2018). Comparative study on composition, physicochemical and antioxidant characteristics of different varieties of kiwifruit seed oil in China. Food Chem. 264, 411-418. doi: 10.1016/j.foodchem.2018.05.063

Deng, J. J., Yang, H. X., Fan, D. D., Cao, W., and Luo, Y. E. (2013). Antibacterial activities of polyphenolic extract from kiwi fruit (Actinidia chinensis Planch.) seeds. J. Pure Appl. Microbio. 7, 491-494.

Deters, A. M., Schröder, K. R., and Hensel, A. (2005). Kiwi fruit (Actinidia chinensis) polysaccharides exert stimulating effects on cell proliferation via enhanced growth factor receptors, energy production, and collagen synthesis of human keratinocytes, fibroblasts, and skin equivalents. J. Cell. Physiol. 202, 717-722. doi: $10.1002 /$ jcp. 20161

Drummond, L. (2013). The composition and nutritional value of kiwifruit. $A d v$. Food Nutr. Res. Chapter 3.68, 33-54. doi: 10.1016/B978-0-12-394294-4.00003-1

Edmunds, S. J., Roy, N. C., Davy, M., Cooney, J. M., Barnett, M. P., Zhu, S., et al. (2012). Effects of kiwifruit extracts on colonic gene and protein expression levels in IL-10 gene-deficient mice. Br. J. Nutr. 108, 113-129. doi: 10.1017/ S0007114511005241

Fang, T. T., Fang, Y., Xu, X. J., He, M. Y., Zhao, Z. Y., Huang, P. X., et al. (2019). Actinidia chinensis Planch. root extract attenuates proliferation and metastasis of hepatocellular carcinoma by inhibiting epithelial-mesenchymal transition. J. Ethnopharm. 231, 474-485. doi: 10.1016/j.jep.2018.11.014

Fiorentino, A., Mastellone, C., D’Abrosca, B., Pacifico, S., Scognamiglio, M., Cefarelli, G., et al. (2009). g-Tocomonoenol: a new vitamin E from kiwi (Actinidia chinensis) fruits. Food Chem. 115, 187-192. doi: 10.1016/j. foodchem.2008.11.094

Flora of China (2007). Actinidia chinensis Planchon Vol. 12. Beijing: Science Press.

Garcia, C. V., Quek, S. Y., Stevenson, R. J., and Winz, R. A. (2012). Kiwifruit flavour: a review. Trends Food Sci. \& Tech. 24, 82-91. doi: 10.1016/j.tifs.2011.08.012

Gerasopoulos, D., and Drogoudi, P. D. (2005). Summer-pruning and preharvest calcium chloride sprays affect storability and low temperature breakdown incidence in kiwifruit. Postharvest Biol. Technol. 36, 303-308. doi: 10.1016/j. postharvbio.2005.01.005

Gerasopoulos, D., Chlioumis, G., and Sfakiotakis, E. (2006). Non-freezing points below zero induce low-temperature breakdown of kiwifruit at harvest. J. Sci. Food Agric. 86, 886-890. doi: 10.1002/jsfa.2429

Goffi, V., Zampella, L., Forniti, R., Petriccione, M., and Botondi, R. (2019). Effects of ozone postharvest treatment on physicochemical and qualitative traits of Actinidia chinensis 'Soreli' during cold storage. J. Sci. Food Agric. 99, 5654-5661. doi: $10.1002 /$ jsfa. 9823
Gu, J., Li, J., Yang, X. D., Shang, Z. W., Wei, H. Q., Jiang, X. J., et al. (2017). Study on inhibition effect of Actinidia chinensis Planch. extracted by chloroform proliferation of HEP-2 Cells. Chin. Arch. Tradi. Chin. Med. 35, 2835-2838. doi: 10.13193/j.issn.1673-7717.2017.11.028

He, G. N., Hu, X. M., Wang, H., Fan, L., and Wang, B. C. (2015a). Chemical constituents in root of Actinidia chinensis Planch. Zhonghua Zhongyiyao Zazhi. $30,498-500$

He, J., Ma, B. Z., Wang, X. X., Liu, F., Qin, W. J., Zhang, X. L., et al. (2015b). Chemical constituents from the root of Actinidia chinensis (II). Zhongguo Yaoxue Zazhi. 50, 1960-1963. doi: 10.11669/cpj.2014.03.005

He, J., Ma, B. Z., Zhao, T., Wang, W., Wei, F. L., Lu, J., et al. (2014). Chemical constituents from the roots of Actinidia chinensis. Zhongguo Yaoxue Zazhi. 49, 184-186. doi: 10.11669/cpj.2014.03.005

He, M. Y., Hou, J. J., Wang, L. Y., Zheng, M. H., Fang, T. T., Wang, X. D., et al. (2017). Actinidia chinensis Planch. root extract inhibits cholesterol metabolism in hepatocellular carcinoma through upregulation of PCSK9. Oncotarget. 8, 42136-42148. doi: 10.18632/oncotarget.15010

Hettihewa, S. K., Hemar, Y., and Rupasinghe, H. P. V. (2018). Flavonoid-rich extract of Actinidia macrosperma (a wild kiwifruit) inhibits angiotensinconverting enzyme in vitro. Food. 7, 146. doi: 10.3390/foods7090146

Henare, S. J. (2016). The nutritional composition of kiwifruit (Actinidia spp.). Nutr. Compos. Fruit Cultivars, Chapter 15, 337-368. doi: 10.1016/ B978-0-12-408117-8.00015-5

Hou, J. J., Wang, L. Y., and Wu, D. J. (2018). The root of Actinidia chinensis inhibits hepatocellular carcinomas cells through LAMB3. Cell Biol. Toxicol. 34, 321332. doi: 10.1007/s10565-017-9416-7

Huang, Z. Y., Li, J., Zhang, J. F., Gao, Y. Y., and Hui, G. H. (2017). Physicochemical properties enhancement of Chinese kiwi fruit (Actinidia chinensis Planch.) via chitosan coating enriched with salicylic acid treatment. J. Food Meas. Charact. 11, 184-191. doi: 10.1007/s11694-016-9385-1

Hwang, J. S., Cho, C. H., Baik, M. Y., Park, S. K., Heo, H. J., Cho, Y. S., et al. (2017). Effects of freeze-drying on antioxidant and anticholinesterase activities in various cultivars of kiwifruit (Actinidia spp.). Food Sci. Biotechnol. 26, 221-228. doi: 10.1007/s10068-017-0030-5

Iwasawa, H., Morita, E., Yui, S., and Yamazaki, M. (2011). Anti-oxidant effects of kiwi fruit in vitro and in vivo. Biol. Pharm. Bull. 34, 128-134. doi: 10.1248/ bpb. 34.128

Ji, Z., and Liang, X. (1985). Chemical constituents of the root of Actinidia chinensis Planch. Yaoxue Xuebao 20, 778-781.

Jiao, Y., Chen, D. L., Fan, M. T., Young, , and Qu, S. (2019). UPLC-QqQ-MS/ MS-based phenolic quantification and antioxidant activity assessment for thinned young kiwifruits. Food Chem. 281, 97-105. doi: 10.1016/j. foodchem.2018.12.062

Kaur, L., Rutherfurd, S. M., Moughan, P. J., Drummond, L., and Boland, M. J. (2010). Actinidin enhances gastric protein digestion as assessed using an in vitro gastric digestion model. J. Agri. Food Chem. 58, 5068-5073. doi: 10.1021/jf903332a

Ku, C. Y., Wang, Y. R., Lin, H. Y., Lu, S. C., and Lin, J. Y. (2015). Corosolic acid inhibits hepatocellular carcinoma cell migration by targeting the VEGFR2/Src/ FAK pathway. PLoS One. 10, e0126725. doi: 10.1371/journal.pone.0126725

Kvesitadze, G. I., Kalandiya, A. G., Papunidze, S. G., and Vanidze, M. R. (2001). Identification and quantification of ascorbic acid in kiwi fruit by highperformance liquid chromatography. Appl. Biochem. Micro. 37, 215-218. doi: 10.1023/A:1002848302873

Lahlou, E. H., Hirai, N., Kamo, T., Tsuda, M., and Ohigashi, H. (2001). Actinidic acid, a new triterpene phytoalexin from unripe kiwi fruit. Biosci. Biotech. Bioch. 65, 480-483. doi: 10.1271/bbb.65.480

Lee, D. E., Shin, B. J., Hur, H. J., Kim, J. H., Kim, J., Kang, N. J., et al. (2010). Quercetin, the active phenolic component in kiwifruit, prevents hydrogen peroxide-induced inhibition of gap-junction intercellular communication. Brit. J. Nutr. 104, 164-170. doi: 10.1017/S0007114510000346

Lee, I., Lee, B. H., Eom, S. H., Oh, C. S., Kang, H., Cho, Y. S., et al. (2015). Antioxidant capacity and protective effects on neuronal PC-12 cells of domestic bred kiwifruit. Korean J. Hortic. Sci. Technol. 33, 259-267. doi: 10.7235/hort.2015.14123

Li, D. X., and Zhu, F. (2017). Physicochemical properties of kiwifruit starch. Food Chem. 220, 129-136. doi: 10.1016/j.foodchem.2016.09.192

Lin, P. F. (1988). Antitumor effect of Actinidia chinensis polysaccharide on murine tumor. Zhonghua Zhong Liu Za Zhi. 10, 441-444. 
Liu, W., and Hui, G. H. (2015). Kiwi fruit (Actinidia chinensis) quality determination based on surface acoustic wave resonator combined with electronic nose. Bioengineered 6, 1-9. doi: 10.1080/21655979.2014.996430

Luan, X., Li, X. J., and Guo, M. M. (2017). Xiangxi kiwifruit seed component and characteristics of kiwifruit seed oil. Chin. Oils Fats. 42, 136-139. doi: 10.3969/j. issn.1003-7969.2017.08.030

Lv, J. P., Wang, L. Y., Shen, H., and Wang, X. D. (2018). Regulatory roles of OASL in lung cancer cell sensitivity to Actinidia chinensis Planch. root extract (acRoots). Cell Biol. Toxicol. 34, 207-218. doi: 10.1007/s10565-018-9422-4

Ma, Q. S., Suo, J. T., Huber, D. J., Dong, X. Q., Han, Y., Zhang, Z. K., et al. (2014). Effect of hot water treatments on chilling injury and expression of a new C-repeat binding factor (CBF) in 'Hongyang' kiwifruit during low temperature storage. Postharvest Biol. Technol. 97, 102-110. doi: 10.1016/j. postharvbio.2014.05.018

Ma, T. T., Sun, X. Y., Zhao, J. M., You, Y. L., Lei, Y. S., Gao, G. T., et al. (2017). Nutrient compositions and antioxidant capacity of kiwifruit (Actinidia) and their relationship with flesh color and commercial value. Food Chem. 218, 294-304. doi: 10.1016/j.foodchem.2016.09.081

McGhie, T. K., and Ainge, G. D. (2002). Color in ruit of the genus Actinidia: carotenoid and chlorophyll compositions. J. Agri. Food Chem. 50, 117-121. doi: $10.1021 /$ jf010677l

Meng, F. C., Cao, J. F., Zhang, Y. T., and Chang, M. Y. (2017). Optimization of extraction technology of pectin from kiwifruit peel and pulp by ultrasoundassisted extraction. J. Green Sci. Technol. 14, 283-285. doi: 10.16663/j.cnki. lskj.2017.14.101

Michaud, J., and Ane-Margail, M. (1977). Analytical study of catechuic tannins. I. Flavanolic oligomers of Actinidia chinensis Planch. Babin, Bull. Soc. Parma Bord. 116, 52-64.

Montefiori, M., McGhie, T. K., Costa, G., and Ferguson, A. R. (2005). Pigments in the fruit of red-fleshed kiwifruit (Actinidia chinensis and Actinidia deliciosa). J. Agric. Food Chem. 53, 9526-9530. doi: 10.1021/jf051629u

Niu, H., Song, D., Sun, Y. L., Zhang, W. X., Mu, H. B., and Duan, J. Y. (2016). Preparation and sulfation of an a-glucan from Actinidia chinensis roots and their potential activities. Int. J. Biol. Macromol. 92, 981-987. doi: 10.1016/j. ijbiomac.2016.07.091

Papunidze, G., Kalandiya, A., Papunidze, S., and Vanidze, M. (2001). Kiwifruit chemical composition. Bull. Ge. Acad. Sci. 164, 544-546.

Qu, L., Liu, Q., Zhang, Q., Tuo, X., Fan, D., Deng, J., et al. (2019). Kiwifruit seed oil prevents obesity by regulating inflammation, thermogenesis, and gut microbiota in high-fat diet-induced obese C57BL/6 mice. Food Chem. Toxicol. 125, 85-94. doi: 10.1016/j.fct.2018.12.046

Sfakiotakis, E., Chlioumis, G., and Gerasopoulos, D. (2005). Preharvest chilling reduces low temperature breakdown incidence of kiwifruit. Postharvest Biol. Technol. 38, 169-174. doi: 10.1016/j.postharvbio.2005.06.010

Sharon, J. H. (2016). The nutritional composition of kiwifruit (Actinidia spp.). Nutr. Compos. Fruit Cultivars. 15, 343-345. doi: 10.1016/ B978-0-12-408117-8.00015-5

Shen, L., Zhang, G. J., Zhang, G. S., Song, W. Y., and Xu, G. H. (2014). Effect of Actinidia chinensis polysaccharide on apoptosis of MFC and their orthotopic transplanted tumor of gastric cancer. Chin. Trad. Herb. Drugs. 45, 673-678. doi: 10.7501/j.issn.0253-2670.2014.05.015

Shu, Q., De Silva, U. M., Chen, S., Peng, W. D., Ahmed, M., Lu, G. J., et al. (2008). Kiwifruit extract enhances markers of innate and acquired immunity in a murine model. Food Agri. Immunol. 19, 149-161. doi: 10.1080/09540100802117198

Sivakumaran, S., Huffman, L., Sivakumaran, S., and Drummond, L. (2018). The nutritional composition of Zespri SunGold kiwifruit and ZespriSweet green kiwifruit. Food Chem. 238, 195-202. doi: 10.1016/j.foodchem.2016.08.118

Sun, L. F., Li, X. F., Li, G., Dai, B., and Tan, W. (2017). Actinidia chinensis Planch. improvestheindices of antioxidant and anti-inflammation status of type 2 diabetes mellitus by activating keap1 and nrf2 via the upregulation of microRNA-424. Oxid. Med. Cell. Longev. 2017, 7038789. doi: 10.1155/2017/7038789

The Plant List (2013). Version 1.1. Published on the internet; http://www. theplantlist.org/ (accessed 1st January).

Twidle, A. M., Barker, D., Seal, A. G., Fedrizzi, B., and Suckling, D. M. (2018). Identification of floral volatiles and pollinator responses in kiwifruit cultivars, Actinidia chinensis var. chinensis. J. Chem. Ecol. 44, 406-415. doi: 10.1007/ s10886-018-0936-2
United Nations Food and Agriculture Organization. (2016). Kiwifruit production in 2016. "Crops/World Regions/Production Quantity from pick lists". Retrieved 2018-02-20.

United States Department of Agriculture USDA Food Composition Databases. (2018). https://ndb.nal.usda.gov/ndb/. Accessed on April, 2018.

Wang, H. X., and Ng, T. B. (2002). Isolation of an antifungal thaumatin-like protein from kiwi fruits. Phytochem. 61, 1-6. doi: 10.1016/S0031-9422(02)00144-9

Wang, M. Y., MacRae, E., Wohlers, M., and Marsh, K. (2011). Changes in volatile production and sensory quality of kiwifruit during fruit maturation in Actinidia deliciosa 'Hayward' and A. chinensis 'Hort16A'. Postharvest Biol. Technol. 59, 16-24. doi: 10.1016/j.postharvbio.2010.08.010

Wang, Q., Lei, X. X., Yang, X. D., Xin, T., and Li, Y. Q. (2017). Purification of total triterpenes from the root of Actinidia chinensis Planch. and its antitumor activity. Guangdong Med. J. 38, 514-518. doi: 10.3969/j.issn.1001-9448.2017.04.005

Wang, Q., Xu, Y. F., Gao, Y., and Wang, Q. (2018). Actinidia chinensis Planch. polysaccharide protects against hypoxia-induced apoptosis of cardiomyocytes in vitro. Mol. Med. Rep. 18, 193-201. doi: 10.3892/mmr.2018.8953

Wang, Y., Zhao, C. L., Li, J. Y., Liang, Y. J., Yang, R. Q., Liu, J. Y., et al. (2018). Evaluation of biochemical components and antioxidant capacity of different kiwifruit (Actinidia spp.) genotypes grown in China. Biotec. Biotechnol. Equip. 32, 1-8. doi: 10.1080/13102818.2018.1443400

Wei, L. B., Ma, S. Y., Liu, H. X., Huang, C. S., and Liao, N. (2018). Cytotoxic triterpenoids from roots of Actinidia chinensis. Chem. Biodivers. 15, e1700454. doi: $10.1002 / c b d v .201700454$

Wurms, K. V., and Cooney, J. M. (2006). Isolation of a new phenolic compound, 3, 5-dihydroxy-2-(methoxycarbonylmethyl) phenyl 3, 4-dihydroxybenzoate, from leaves of Actinidia chinensis (kiwifruit). Asian J. Biochem. 1, 325-332. doi: 10.3923/ajb.2006.325.332

Xia, C., Chen, J., Zhang, Y. J., Zhu, Y. Q., Li, H. J., and Deng, J. L. (2017). Isolation, purification, monosaccharide composition and anticancer proliferation activity of polysaccharide fraction from Hongyang kiwifruit (Actinidia chinensis). Food Sci. 38, 126-131. doi: 10.7506/spkx1002-6630-201721020

Xie, Z. W. (1975). National compilation of Chinese herbal medicine Vol. I. Beijing: People's Health Publishing House, 820-821.

Xu, Y. X., Xiang, Z. B., Jin, Y. S., Shen, Y., and Chen, H. S. (2010). Two new triterpenoids from the roots of Actinidia chinensis. Fitoterapia. 81, 920-924. doi: 10.1016/j.fitote.2010.06.007

Xu, Y. X., Xiang, Z. B., Jin, Y. S., Xu, W., Sun, L. N., Chen, W. S., et al. (2016). Constituents from the roots of Actinidia chinensis and their cytochrome P450 enzyme inhibitory activities. Chem. Biodivers. 13, 1454-1459. doi: 10.1002/ cbdv. 201500518

Yang, H. L., Lee, Y. C., Han, K. S., Singh, H. S., Yoon, M., Park, J. H., et al. (2013). Green and gold kiwifruit peel ethanol extracts potentiate pentobarbitalinduced sleep in mice via a GABAergic mechanism. Food Chem. 136, 160-163. doi: 10.1016/j.foodchem.2012.07.111

Yang, Q., Rao, J., Yi, S., Meng, K., Wu, J., and Hou, Y. (2013). Antioxidant enzyme activity and chilling injury during low-temperature storage of kiwifruit cv Hongyang exposed to gradual postharvest cooling. Horticul. Environ. Biotechnol. 53, 505-512. doi: 10.1007/s13580-012-0101-8

Yang, Q., Wang, F., and Rao, J. (2016). Effect of putrescine treatment on chilling injury, fatty acid composition and antioxidant system in kiwifruit. PLoS One. 11, e0162159. doi: 10.1371/journal.pone.0162159

Yu, A. N., Tian, D. T., Qu, W. Y., Tan, Z. W., and Song, X. J. (2009). Chemical composition of the essential oil of the roots of Actinidia chinensis from China. Chem. Nat. Com. 45, 108-109. doi: 10.1007/s10600-009-9235-z

Yuliarti, O., Goh, K. K., Matia-Merino, L., Mawson, J., and Brennan, C. (2015a). Extraction and characterisation of pomace pectin from gold kiwifruit (Actinidia chinensis). Food Chem. 187, 290-296. doi: 10.1016/j.foodchem.2015.03.148

Yuliarti, O., Goh, K. K., Matia-Merino, L., Mawson, J., Drummond, L., and Brennan, C. S. (2008). Effect of extraction techniques and conditions on the physicochemical properties of the water soluble polysaccharides from gold kiwifruit (Actinidia chinensis). Inter. J. Food Sci. Tec. 43, 2268-2277. doi: 10.1111/j.1365-2621.2008.01866.x

Yuliarti, O., Matia-Merino, L., Goh, K. K., Mawson, J., Williams, M. A., and Brennan, C. (2015b). Characterization of gold kiwifruit pectin from fruit of different maturities and extraction methods. Food Chem. 166, 479-485. doi: 10.1016/j.foodchem.2014.06.055 
Zhang, L., Zhang, W. X., Wang, Q. J., Wang, D. D., Dong, D. Q., Mu, H. B., et al. (2015). Purification, antioxidant and immunological activities of polysaccharides from Actinidia Chinensis roots. Int. J. Biol. Macromol. 72, 975983. doi: 10.1016/j.ijbiomac.2014.09.056

Zhang, T., Li, C., Luo, A. W., Tang, M. L., Chen, H., Li, X. L., et al. (2016). Antioxidant activities in vitro of different fruit parts of eight kiwifruit varieties. Food Sci. 37, 88-93. doi: 10.7506/spkx1002-6630-201619015

Zhang, X. Y., Zhou, Y., Wei, Z. P., Shen, J., Wang, L. K., Ma, Z. Q., et al. (2018). Antiphytoviral toxins of Actinidia chinensis root bark (ACRB) extract: laboratory and semi-field trials. Pest Manag. Sci. 74, 1630-1636. doi: 10.1002/ps.4854

Zhao, T., He, J., Wang, X., Ma, B., Wang, X., Zhang, L., et al. (2014). Rapid detection and characterization of major phenolic compounds in radix Actinidia chinensis Planch. by ultra-performance liquid chromatography tandem mass spectrometry. J. Pharm. Biomed. Anal. 98, 311-320. doi: 10.1016/j.jpba.2014.05.019

Zhou, X. F., Liu, Y. H., Tang, L., Zhang, P., and Wu, J. Z. (2010). Chemical constituents from the roots of Actinidia chinensis. Chem. Nat. Compd. 46, 308309. doi: 10.1007/s10600-010-9599-0

Zhou, X. F., Zhang, P., Pi, H. F., Zhang, Y. H., Ruan, H. L., Wang, H., et al. (2009). Triterpenoids from the roots of Actinidia chinensis. Chem. Biodivers. 6, 12021207. doi: $10.1002 / \mathrm{cbdv} .200800214$
Zhu, W. J., Yu, D. H., Zhao, M., Lin, M. G., Lu, Q., Wang, Q. W., et al. (2013). Antiangiogenic triterpenes isolated from Chinese herbal medicine Actinidia chinensis Planch. Anticancer Ag. Med. Chem. 13, 195-198. doi: 10.2174/1871520611313020002

Zuo, L. L., Wang, Z. Y., Fan, Z. L., Tian, S. Q., and Liu, J. R. (2012). Evaluation of antioxidant and antiproliferative properties of three Actinidia (Actinidia kolomikta, Actinidia arguta, Actinidia chinensis) extracts in vitro. Inter. J. Mol. Sci. 13, 5506-5518. doi: 10.3390/ijms13055506

Conflict of Interest: The authors declare that the research was conducted in the absence of any commercial or financial relationships that could be construed as a potential conflict of interest.

Copyright (c) 2019 He, Fang, Chen, Zhao, Li, Meng and Huang. This is an open-access article distributed under the terms of the Creative Commons Attribution License (CC $B Y)$. The use, distribution or reproduction in other forums is permitted, provided the original author(s) and the copyright owner(s) are credited and that the original publication in this journal is cited, in accordance with accepted academic practice. No use, distribution or reproduction is permitted which does not comply with these terms. 